Canadian University Music Review

Revue de musique des universités canadiennes

\title{
Chanter le Ring
}

Un entretien de Jeffrey Tate avec Jean-Jacques Nattiez

\section{Jeffrey Tate}

Numéro 8, 1987

URI : https://id.erudit.org/iderudit/1014935ar

DOI : https://doi.org/10.7202/1014935ar

Aller au sommaire du numéro

Éditeur(s)

Canadian University Music Society / Société de musique des universités canadiennes

ISSN

0710-0353 (imprimé)

2291-2436 (numérique)

Découvrir la revue

Citer ce document

Tate, J. (1987). Chanter le Ring : un entretien de Jeffrey Tate avec Jean-Jacques Nattiez. Canadian University Music Review / Revue de musique des universités canadiennes, (8), 78-133. https://doi.org/10.7202/1014935ar

All Rights Reserved @ Canadian University Music Society / Société de musique des universités canadiennes, 1986
Ce document est protégé par la loi sur le droit d'auteur. L'utilisation des services d’Érudit (y compris la reproduction) est assujettie à sa politique d'utilisation que vous pouvez consulter en ligne.

https://apropos.erudit.org/fr/usagers/politique-dutilisation/ 


\section{CHANTER LE RING}

\section{Un entretien de Jeffrey Tate avec Jean-Jacques Nattiez}

En juillet 1986, dix ans se seront écoulés depuis qu'a éclaté à Bayreuth, la "bombe" de la Tétralogie dite du Centenaire, mise en scène par Patrice Chéreau et dirigée par Pierre Boulez. Depuis, des millions de téléspectateurs ont vu cette production au petit écran. Pour la préparation de notre livre, Tétralogies (Wagner, Boulez, Chéreau), essai sur l'infidélité (Nattiez 1983), nous avions procédé à une longue interview de Boulez qui a fait l'objet d'une publication à tirage très restreint (Boulez-Tate-Nattiez 1982) et dont les éléments essentiels ont été repris dans le chapitre du livre consacré à la partie musicale de cette production. Nous avions aussi interviewé, à Bayreuth en juillet 1980 et à New York en mars 1981, Jeffrey Tate, l'assistant de Pierre Boulez, celui qui, en fait, a assumé la préparation du travail des chanteurs et des chanteuses.

Jeffrey Tate était alors inconnu du grand public. Accompagnateur au Covent Garden après des études complètes de médecine, il avait seulement dirigé une Flûte enchantée en Suède et une Carmen à Kassel. Mais après le Ring, Boulez demande à Tate d'être le répétiteur des chanteurs pour la création de la version intégrale de Lulu à l'Opéra de Paris en 1979. C'est avec cette oeuvre qu'il fait ses débuts comme chef d'orchestre au Metropolitan Opera en décembre 1980. Et depuis, nous assistons à l'envolée d'une fulgurante carrière: Don Juan et Wozzeck au Met, Parsifal à Nice, Ariane à Naxos à Paris, L'Or du Rhin à Genève. Entre 1982 et 1985, il aura dirigé pas moins de vingt-trois opéras, dont une création de Henze au festival de Salzbourg, en même temps qu'il aura entrepris des disques et un certain nombre de concerts symphoniques dont trois à Montréal en avril 1985. Demain, le Philharmonique de Berlin. Bientôt, il partagera la direction artistique de Covent Garden avec Bernard Haitink. Et Bayreuth, sûrement, un jour...

On possède fort peu de témoignages de première main et de documents précis sur la conception et la technique des grandes interprétations. L'émergence de ce nouveau talent nous paraît justifier que nous donnions à cet entretien avec Jeffrey Tate une diffusion plus vaste que le rapport de recherches où il était confiné (Boulez-Tate-Nattiez 1982 : 210-270). Jeffrey Tate nous explique ici comment il a fait travailler les "chantacteurs" du Ring de Bayreuth entre 1976 et 1980 : Gwyneth Jones (Brünnhilde), Manfred Jung (Siegfried), Heinz Zednik (Loge, Mime), Donald McIntyre (Wotan) et bien d'autres... Ecoutons-le. 
Jean-Jacques Nattiez (J.J.N.) : Jeffrey Tate, vous êtes un homme particulièrement modeste, mais je crois que c'est justice que de vous associer pleinement aux quatre noms - Boulez, Chéreau, Peduzzi, Schmidt 1 - à qui nous devons cette Tétralogie désormais historique : c'est vous qui avez assumé quasi totalement la préparation des chanteurs, et ce n'est sans doute pas pour rien que Boulez, fait unique dans les annales de Bayreuth et de beaucoup d'opéras, vous a donné la possibilité de saluer le public avec lui lors de la dernière du Crépuscule en 1980. Racontez-nous comment s'est passé tout ce travail à Bayreuth.

Jeffrey Tate (J.T.) : En 1976, lors de la première année, Boulez entrait pour la première fois dans cette oeuvre. Il y avait des répétitions avec les chanteurs, j'accompagnais au piano et il était là. Ce qu'il voulait, c'était donner la grande architecture, aux chanteurs et à moi-même. Il a indiqué les couleurs, les expressions, les tempi, tels qu'il les concevait à l'époque et j'ai tout noté dans ma partition. Après cela, quand j'ai compris exactement ce qu'il voulait, c'est-à-dire l'esprit de ce qu'il voulait, il m'a laissé le travail avec les chanteurs.

J.J.N. : Quel était votre rôle vis-à-vis de lui, au début ?

J.T. J'ai travaillé comme répétiteur au Covent Garden pour les Ring de Georg Solti et Colin Davis. Boulez voulait avoir mon opinion, en particulier pour des détails pratiques. Par exemple, il s'interrogeait sur la façon de réaliser certaines transitions : fallait-il battre à deux, à quatre temps ? J'avais beaucoup discuté de cela avec Davis et j'ai transmis ces informations à Boulez, non pas pour qu'il refasse systématiquement la même chose, mais simplement pour lui communiquer mon expérience de ces passages. Bien sûr, on a discuté de tout cela seuls. Devant les chanteurs, lorsqu'il était là, j'intervenais peu. Puisqu'il s'agissait, avec lui, de la structure d'ensemble, il n'était pas question d'arrêter les chanteurs trop souvent, avec des petits détails. Je notais ou mémorisais les erreurs ou les problèmes et j'en parlais au

1 Richard Peduzzi et Jacques Schmidt sont respectivement le décorateur et le costumier habituels de Patrice Chéreau. 
chanteur après. Boulez savait que je comprendrais très bien ce qu'il voulait, et il me laissait régler les choses avec les artistes. A partir de la seconde année, il m'a confié entièrement la direction de la partie vocale.

\section{J.J.N. : Vous en êtes satisfait?}

J.T. : J'aime beaucoup cette division du travail. J'ai plus de contact avec les chanteurs et je vais à toutes les répétitions. Lui peut ainsi se concentrer sur l'orchestre, et vous avez vu quelle entreprise de décapage cela signifiait. Sauf lorsqu'il y a eu des crises sérieuses, assez rarement je dois dire, il m'a laissé la responsabilité avec les chanteurs.

\section{J.J.N. : Intervenez-vous pour l'orchestre?}

J.T. : Jamais pendant les répétitions, évidemment. J'ai noté, surtout les deux premières années, des petites fautes que je lui communiquais ensuite. L'essentiel, c'était que j'aille dans la salle écouter comment ça sonnait, parce que, évidemment, il n'a jamais pu le faire. On a beaucoup discuté des problèmes de balance. J'ai été son oreille dans la salle.

J.J.N. : En vous écoutant, on a le sentiment que quelque chose vous prédisposait d'entrée de jeu à accepter la conception boulézienne de ce Ring.

J.T. : Oui. Mon expérience de l'Anneau est anglaise. Certes, elle est différente de ce que veut Boulez. Les tempi anglais, en particulier, sont plus lents que les siens, mais tout de même, notre conception est plus proche de celle de Boulez que de celle des Allemands. Par exemple, il y a un vieux chef anglais, Gudol - il a été l'assistant de Furtwängler - qui a fait des Ring passablement excentriques et extraordinaires, en tout cas très intéressants. Et il a exerceé une influence profonde sur Colin Davis. Ce chef lui avait dit : "L'essentiel, c'est de jouer toutes les notes, de tout entendre." Lorsque j'ai vu Boulez à Londres pour la première fois, il m'a dit : "La chose la plus importante, c'est de tout jouer. Et c'est capital pour les tempi, car même dans des tempi rapides, avec du travail, on peut tout entendre et obtenir la clarté". Je me suis 
tout de suite senti en accord avec cette conception. J'ai horreur de l'Anneau quand la force et les effets dominent tout. La partition n'est pas comme ça ; au contraire, c'est quelque chose d'un grand raffinement.

J.J.N. : Avez-vous le sentiment que Boulez ait modifié sa conception au cours des années?

J.T. : En 1976, il avait déjà, fondamentalement, cette volonté d'obtenir la clarté des détails et la grande architecture, mais ça n'était pas réussi comme aujourd'hui, et je ne crois pas que ce soit lui adresser une critique que de dire combien il a progressé.

J.J.N. : Il le reconnaît lui-même !

J.T. : Sur beaucoup d'aspects il a changé d'avis entre 1976 et 1977 et nous nous sommes rencontrés à Paris pour en discuter. A partir de 1979, les choses sont devenues plus stables. Mais c'est une oeuvre tellement complexe que, même aujourd'hui, il reste des points d'interrogation.

J.J.N. : Et j'imagine que la rencontre de sa conception musicale avec la mise en scène de Chéreau a pu aussi le faire bouger ?

J.T. : La première année, ils n'avaient pas toujours la même idée pour un même passage....

J.J.N. : ...Chéreau invente sur le tas, avec la matière première vivante devant lui, avec l'acteur....

J.T. : Oui, il ne faut pas croire qu'ils puissent se mettre d'accord par avance, sur le papier, quant à la conception de chaque passage en particulier. Chéreau cherchait dans les mots et Boulez, dans la musique. Le plus souvent, il y a eu rencontre. Par exemple, dans la scène des filles du Rhin du Crépuscule, tous deux ont senti une espèce de nostalgie, de tristesse. Boulez l'a demandé musicalement et Chéreau scéniquement : on n'a rien changé. Je me souviens que, dans la scène 
entre Brünnhilde et Waltraute du même Crépuscule, Boulez voulait que le "Was ist's mit den ewigen Göttern"2 de Brünnhilde ne soit pas trop violent. Il souhaitait une certaine tendresse à l'égard de Wotan, alors que Chéreau attendait un peu d'agacement. C'est le genre de détails où il a pu y avoir divergence.

J.J.N. : Et peut-on parler d'une influence de la conception de Boulez sur celle de Chéreau?

J.T. : Ce n'est pas une règle, loin de là, mais c'est arrivé. Par exemple, à mon sens, la scène de Erda, dans $L ' O r d u$ Rhin, telle que nous la voyons, découle de la musique de ce passage telle que Boulez l'entend. Ce n'est plus la femme solennelle, l'espèce de déesse avec de grands gestes tragiques, qui domine tout de sa voix de poitrine. Non, c'est l'inverse. Bien sûr, elle a un côté Tyrésias, mais cette prophétesse est avant tout une femme, une vieille femme qui parle, avec une voix très belle, mais très simple et presque triste : c'est de la musique de chambre qui nous ramène très loin dans le passé. D'abord, "Weiche, Wotan! weiche!"3 :

2 “Qu'est-il arrivé aux Dieux éternels ?" Nous empruntons la traduction des citations allemandes à la version du Ring de Jean d'Arièges publiée en présentation bilingue par Aubier-Flammarion.

3 "Cède, Wotan, cède !" 


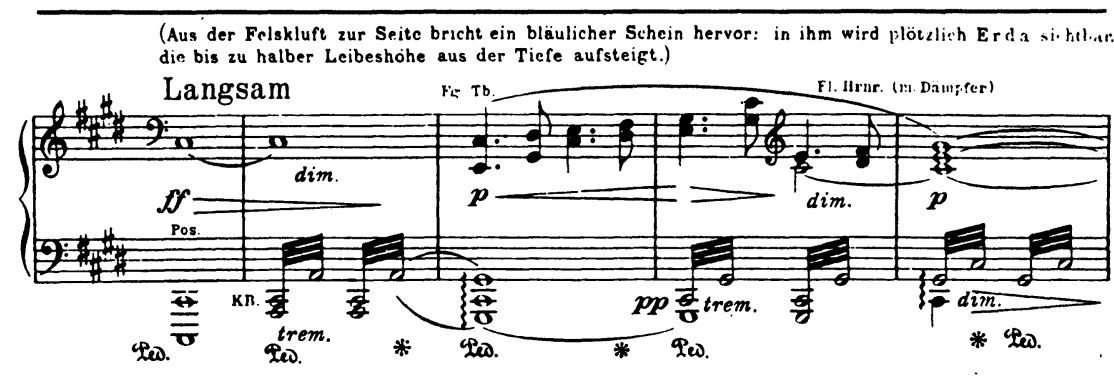

B.Sehr langsam und feierlich
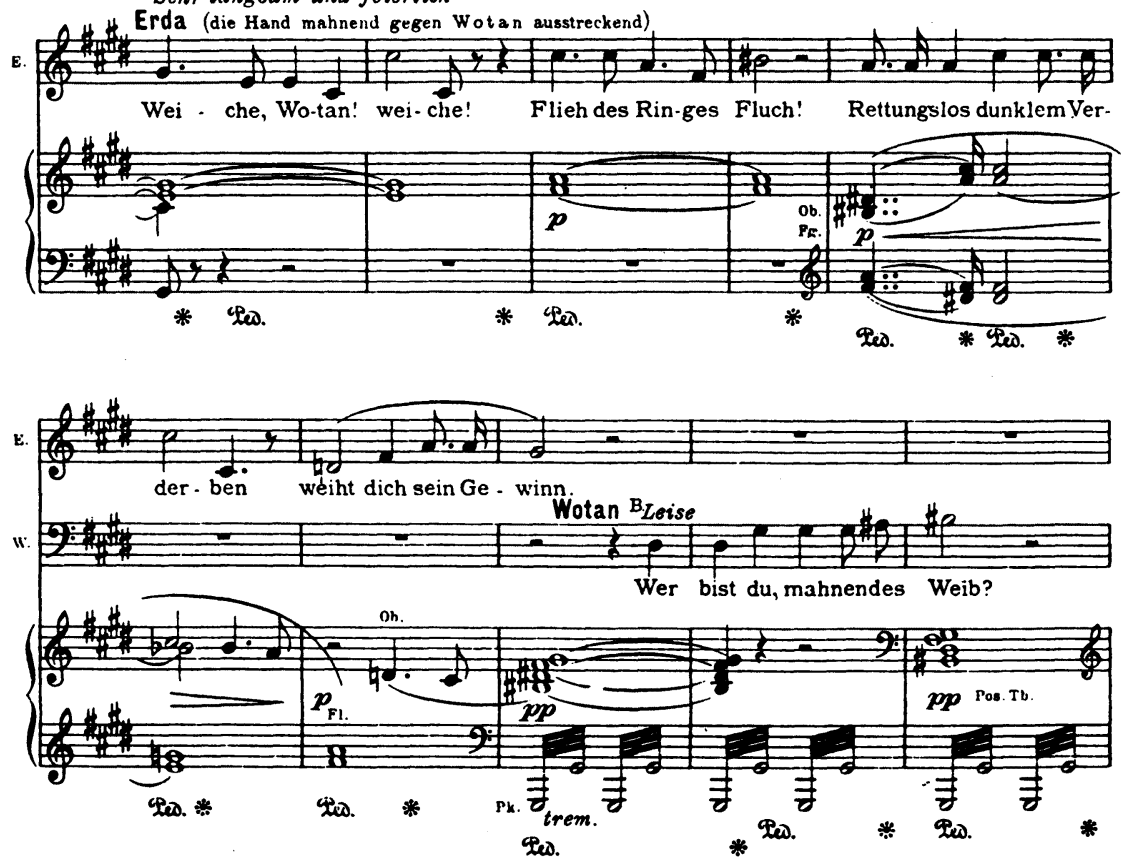

Figure 1. : O.R., IV, $85-86^{4}$

4 Les abréviations O.R., W., S., et C. D. renvoient respectivement à $L^{\prime} O r d u$ Rhin, La Walkyrie, Siegfried, et Le Crépuscule des Dieux. Pour O. R., le 
Habituellement, c'est chanté très fort, très marcato, comme une trompette ou un trombone, comme s'il s'agissait de réveiller un mort ! Non, il doit y avoir de la douceur, de la sympathie. Bien sûr, elle le menace, mais comme on menace un ami. Il ne s'agit pas d'une menace universelle : elle ne s'adresse qu'à lui. Sur le "weiht dich sein Gewinn", 5 il y a un legato, tandis que tout l'orchestre, depuis le début, est piano : cela indique que la relation n'existe qu'entre elle et lui. Il n'y a rien pour les autres, ici. Plus loin, je voudrais signaler un détail. D'abord, les trois "Höre!"6

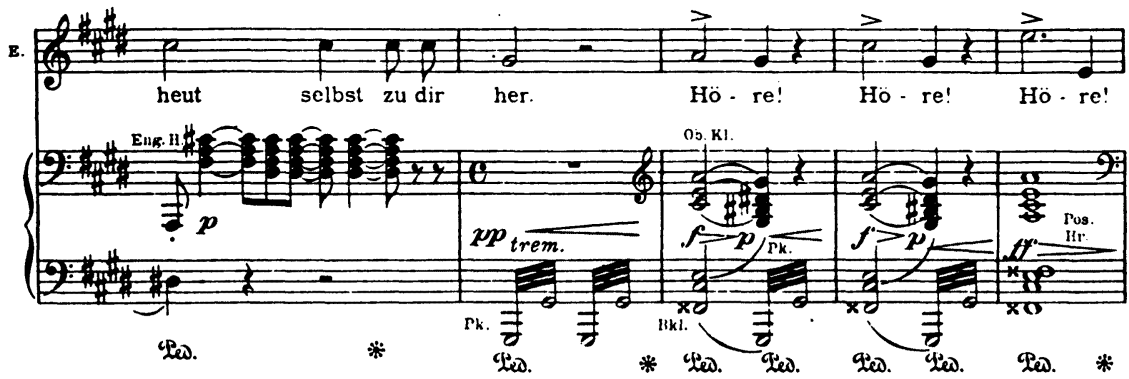

Figure 2. : O.R., IV, 224

Boulez voulait que chacun d'eux soit progressivement plus intense, avec l'ensemble de la dynamique qui augmente. Bien sûr, c'est naturel parce que l'ensemble de la tessiture monte aussi, mais il tenait à ce qu'on le

chiffre romain renvoie à la scène; pour les trois autres opéras, à l'acte. Le nombre qui suit envoie à la page de la réduction pour piano dans l'édition Peters, qui est celle qu'on utilise à Bayreuth.

5 "[à une sombre perdition] te voue son acquisition".

6 "Ecoute !" 
souligne. Pour différencier ces trois mots identiques. Puis, le "alles, was ist, endet!"

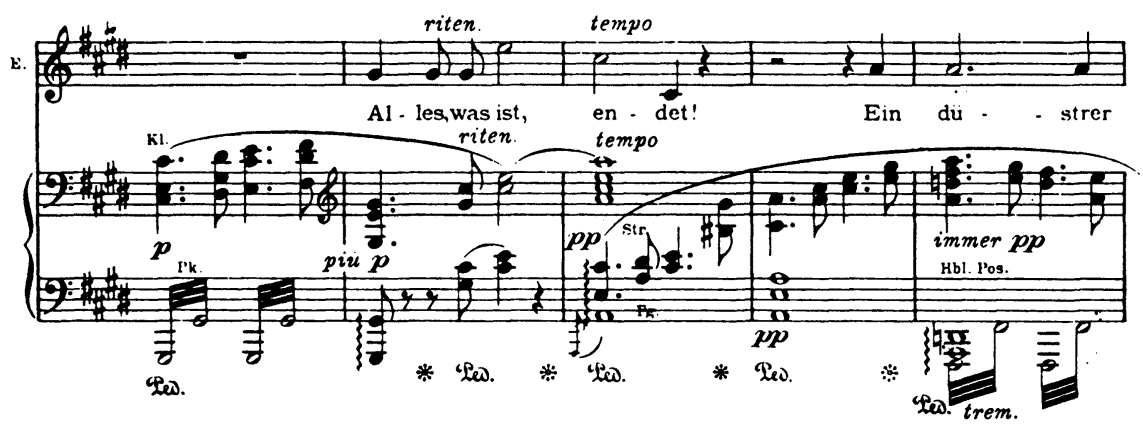

Figure 3. : O.R., IV, 224

Ca ne doit pas être une grande déclaration philosophique. Il faut rester dans le piano, avoir un piano subito qui perturbe et émeuve plus qu'il n'en impose. Ensuite, pour le "Ein düstrer Tag dämmert den Göttern," 8 je demande qu'elle colore un peu les voyelles $\ddot{u}$ et $\ddot{a}:$ c'est $^{2}$ le moment de la confidence. Généralement, le 'dir rat ich, meide den Ring!'9 est chanté très fort. Ici, Boulez a demandé à ce qu'on fasse au contraire un diminuendo :

7 "Tout ce qui est prend fin."

8 "Un sombre jour se lève pour les Dieux.eq.

9 'Je te le conseille, fuis l'anneau !' 


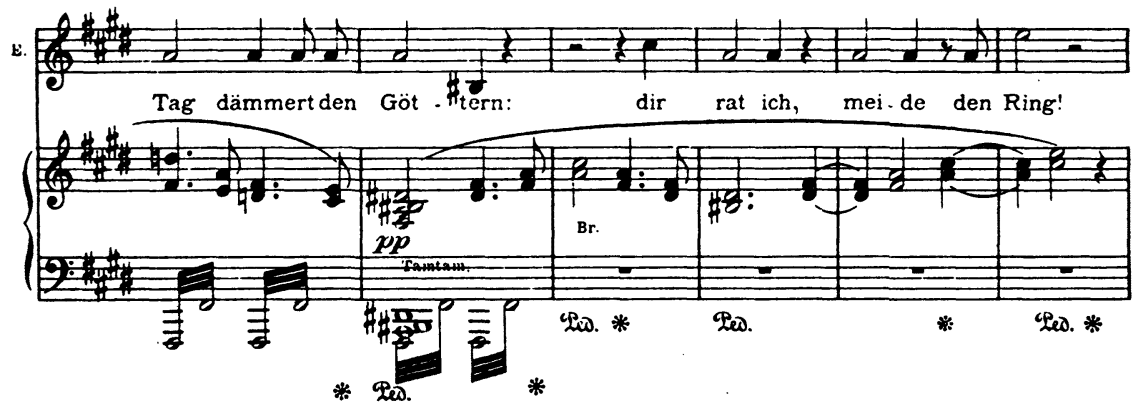

Figure 4. : O.R., IV, 224

Elle parle comme à l'oreille d'un enfant. C'est la musique qui parle, pas les mots. Si on veut $\mathrm{y}$ voir quelque chose de très grave, on peut chanter forte, mais c'est pianissimo à l'orchestre, et le mot 'Ring' doit être tout à fait piano. Après ce mot, il y a un petit pizzicato, comme ça, presque rien... Bien sûr, il y a un point d'exclamation, mais c'est un point d'exclamation intérieur. Chéreau a entendu Erda comme cela, et il a parfaitement compris ce qu'il pouvait en tirer pour la mise en scène.

Weigel chante Erda sur toutes les scènes du monde, mais Bayreuth est le seul endroit où elle le joue dans ce style. Voilà un effet vocal qui a été inspiré par Boulez, avec sa façon très claire de voir - si j'ose dire - quelque chose de nouveau qui, pourtant, existe dans la partition. Et ma tâche, précisément, consiste à maintenir, d'une année à l'autre, cette interprétation.

\section{J.J.N. : C'est difficile?}

J.T. : Oui, parce que les chanteurs oublient ce qu'on leur dit et reprennent les 'mauvaises' habitudes... 
J.J.N. : Chéreau s'en est beaucoup plaint pour la mise en scène également !

J.T. : Et vous savez que tout ce Ring est particulièrement exigeant : il $\mathrm{y}$ a tout d'abord la question des tempi de Boulez qu'on a tendance à relâcher d'une répétition à l'autre. ${ }^{10} \mathrm{La}$ mise en scène absorbe tellement le chanteur qu'il lui est parfois difficile de maintenir son attention à la fois sur la tenue de scène et l'expression vocale.

J.J.N. : D'autant plus que, on l'a vu, les tempi de Boulez dans les transitions ne sont pas évidents.

J.T. : En effet, on ne peut noter la manière d'exécuter une transition, et quand les chanteurs ont appris leur rôle, ils ne savaient pas ce que Boulez ferait. Voyez dans la seconde scène de L'Or du Rhin :

10 Là-dessus, cf. Nattiez $1983: 220-231$. 

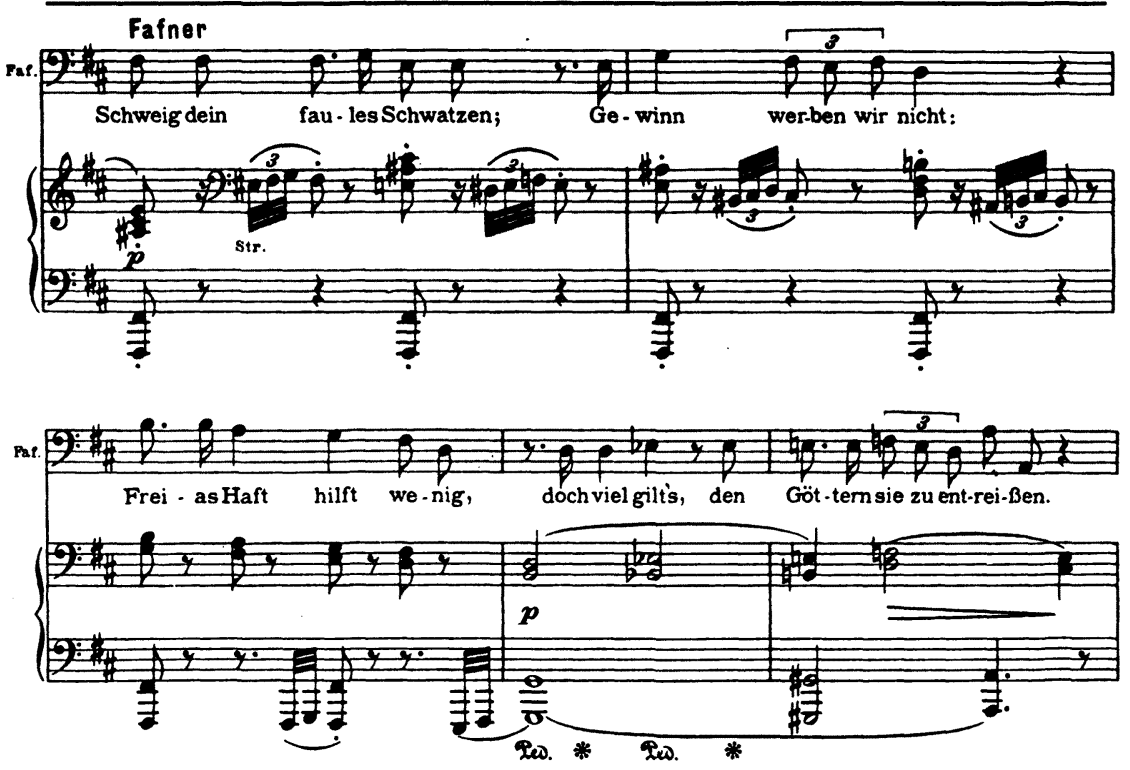

Figure 5. : O.R., II, 85-86

Sur 'Schweig dein faules Schwatzen', ${ }^{11}$ Boulez maintient le tempo pendant deux mesures, mais à partir de 'Freias Haft...', il commence un ritardando qui n'est pas naturel pour l'interprète. Ce sont là des choses que Boulez a souvent trouvées au cours des répétitions. Mon travail consiste ensuite à les leur rappeler constamment.

Il y a aussi les obstacles simplement techniques de cette mise en scène. Les fameux nuages de Chéreau, pendant la première scène de $L^{\prime} O r d u$ Rhin : au tout début de la scène, c'est très difficile d'obtenir le vrai tempo, même si Boulez fait de grands gestes. 'Des Goldes Schlaf/hütet ihr schlecht!'12

11 'Cesse ton vain bavardage.'

12 'Sur le sommeil de l'or/vous veillez mal.' 

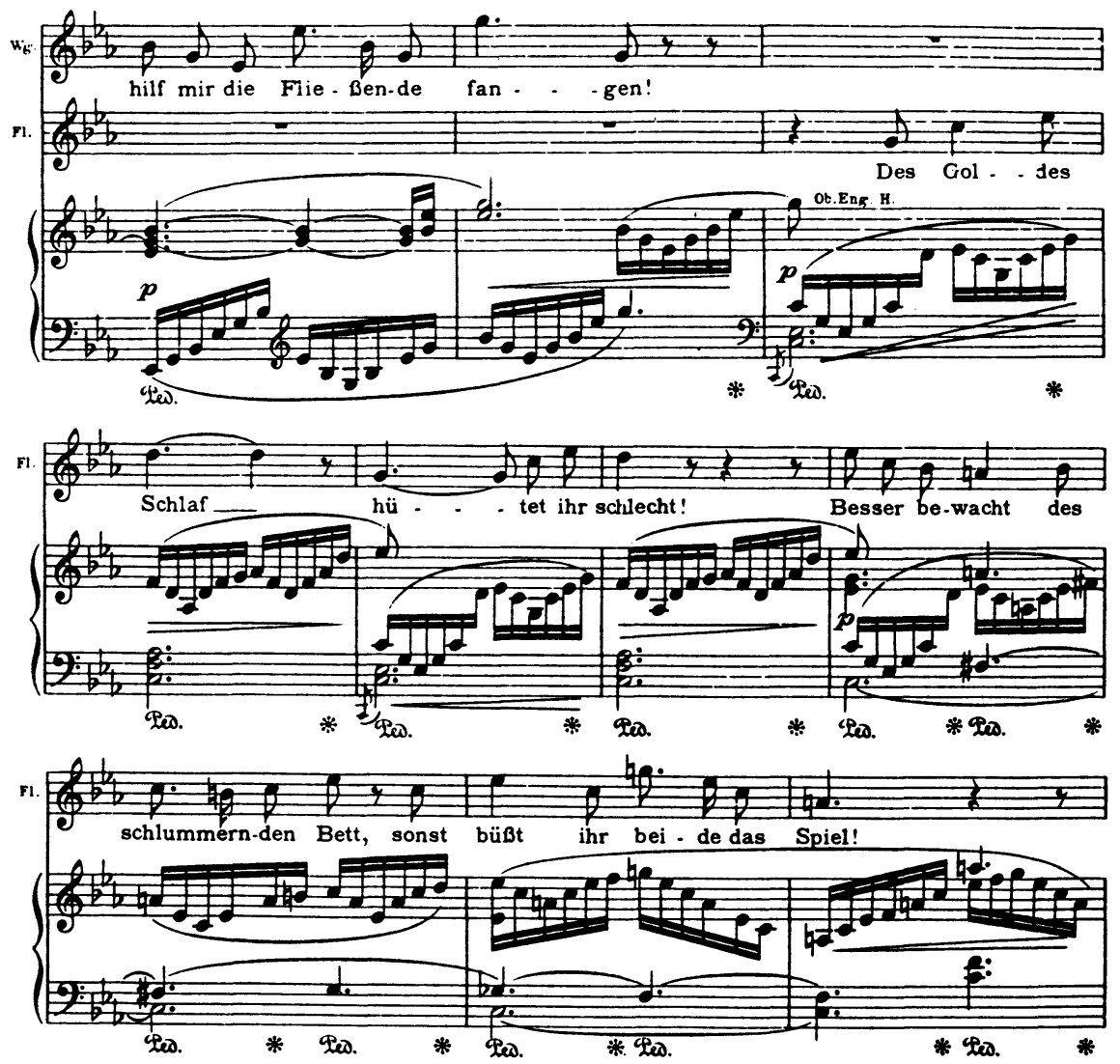

Figure 6. : O.R., I, 11

Les mots exigent quelque chose de plus pesant. Flosshilde fait des reproches aux autres filles : 'Ca n'est pas bien, ce que vous faites là !' 
Je demande une insistance sur le sch de 'schlecht', un marcato sur 'bewacht', puis sur 'beide'. Or je veux l'expression, Boulez son tempo et Chéreau son brouillard !!

Même avec des télévisions tout autour de la scène, ça n'a pas été facile : pour le rôle d'Alberich, dans la scène du Nibelheim, au premier acte de Siegfried. Dans la scène des filles du Rhin du Crépuscule, Chéreau les fait ramper. Boulez ne voyait que les têtes et les interprètes avaient de la peine à voir la battue. De plus, il leur était difficile de s'entendre entre elles. Or, il $\mathrm{y}$ a là un gros problème d'équilibre sonore : la deuxième fille était souvent avancée sur la scène par rapport à la première. Du point de vue de l'expression, nous voulions un legato qui traduise la nostalgie et la tristesse de ces filles. La seule façon d'y arriver, c'est d'avoir un entraînement tel qu'on oublie les cordes vocales, le brouillard et Boulez! Je dois dire que, lorsqu'elles y sont parvenues, c'était merveilleux. Et tout cela, pour ne rien dire des pauvres Walkyries qui traînaient, d'année en année, des figurants de plus en plus grands.

L'objectif de l'entraînement était donc d'obtenir une énonciation vocale aussi naturelle que possible : pour des raisons pratiques, on vient de le voir. Mais aussi pour des raisons esthétiques : Chéreau, Boulez et moi, nous voulions un Ring qui n'ait pas la lourdeur mythologique du Wagner XIXe siècle, mais un Ring où la façon de chanter fasse corps avec la mise en scène.

J.J.N. : Dans l'ensemble, vos 'chantacteurs' ont-ils réussi à accorder autant d'attention aux exigences musicales qu'aux exigences scéniques?

J.T. : Plus ou moins. Je dois dire que je n'ai pas toujours réussi à obtenir, surtout de la part des rôles les plus lourds, la justesse d'intonation souhaitable. Tel ténor n'arrive pas à maintenir les aigus, et il presse le tempo contre l'avis de Boulez. Là, c'est une soprano qui traîne dans l'émission des consonnes. Le vrai problème, c'est que malgré les conditions de travail à Bayreuth, les six semaines de répétition par année ne sont pas suffisantes. 
En fait, j'ai affaire à deux situations. Avec les artistes qui connaissent déjà le rôle, comme Jones (Brünnhilde) ou McIntyre (Wotan), il s'agissait surtout de les convaincre d'adopter les tempi de Boulez et de suivre mes indications allant dans le sens de l'expression voulue par Chéreau. Il fallait donc, avec eux, effacer les habitudes anciennes. Avec les nouveaux venus, et ils étaient assez nombreux, j'ai opéré un travail normal de répétiteur ; d'abord apprendre la musique, les paroles et se pénétrer de leur signification, puis trouver avec eux le bon phrasé, les couleurs et les dynamiques correspondant aux intentions de Chéreau.

J.J.N. : Dans le détail, c'est donc vous qui avez trouvé la traduction vocale de l'expression demandée par le metteur en scène?

J.T. : La plupart du temps, oui. Je dis la plupart du temps, car je ne me considère pas responsable des trouvailles de Zednik (le Loge de $L^{\prime} O r$ $d u$ Rhin et le Mime de Siegfried). Zednik a peut-être une seule couleur dans la voix, mais comme il sait rendre le texte avec une clarté particulière, et un grand soin dans l'expression, on a l'impression qu'il offre beaucoup de couleurs dans sa voix. Loge est le seul personnage qui voit clair dès le début, dans ce Ring...

J.J.N. : Oui, Brünnhilde doit attendre la fin pour vraiment réaliser...

J.T. : ...et ça s'entend précisément dans la clarté de la voix de Zednik. Dans son cas, donc, les choses sont plus venues de lui que de moi.

J.J.N. : Et sinon?

J.T. : La mise en scène de Chéreau, tout comme l'interprétation musicale de Boulez, se caractérise par une attention extraordinaire au détail. Par goût personnel, parce que je conçois ce Ring comme une marquetterie musicale tout en finesse, je n'ai eu aucune peine à trouver cet équivalent vocal dans une attention spéciale aux variations de couleur, d'intensité et de dynamiques. Je n'ai pas hésité à contraster des moments tantôt très piano ou très forte, très hâchés ou très legato. Et évidemment, c'était plus facile avec des chanteurs qui abordaient le rôle pour la première fois. 
J.J.N. : Vous avez discuté de tout cela avec Chéreau ?

J.T. : Vous savez, le problème était le même entre Chéreau et moi qu'entre Chéreau et Boulez. Chéreau est un homme pratique et il ne va pas me dire avant : à tel endroit, je vais faire cela, à tel autre, telle autre chose. Ca dépend du personnage, de l'acteur, de ce qui arrive lors de la première répétition. J'ai donc surtout déduit de ce qui se passait ce que je devais demander vocalement aux chanteurs. Ca n'a jamais été aride mais au contraire quelque chose de très vivant. On a travaillé dans l'atmosphère d'improvisation qui est celle du théâtre parlé. On n'y est pas habitué sur les scènes lyriques, mais c'est sans doute parce que Chéreau y a apporté ces exigences et ces modalités de travail que ce Ring est, entre autres raisons, quelque chose d'exceptionnel.

J.J.N. : Passons donc à votre responsabilité d'interprète proprement dit. On a vu que Boulez essayait se suivre la partition de très près. Est-ce que, pour les voix, elle vous fournit beaucoup d'indications?

J.T. : Pas vraiment. Jusqu'au début du XXe siècle, jusqu'à Richard Strauss en fait, c'est une tradition de la part des compositeurs de ne rien indiquer de particulier pour les chanteurs : il est entendu qu'ils suivront les dynamiques de l'orchestre : piano quand l'orchestre joue piano, legato quand l'orchestre est legato, etc. De ce point du vue, Wagner reste un classique. Ici, par exemple : 


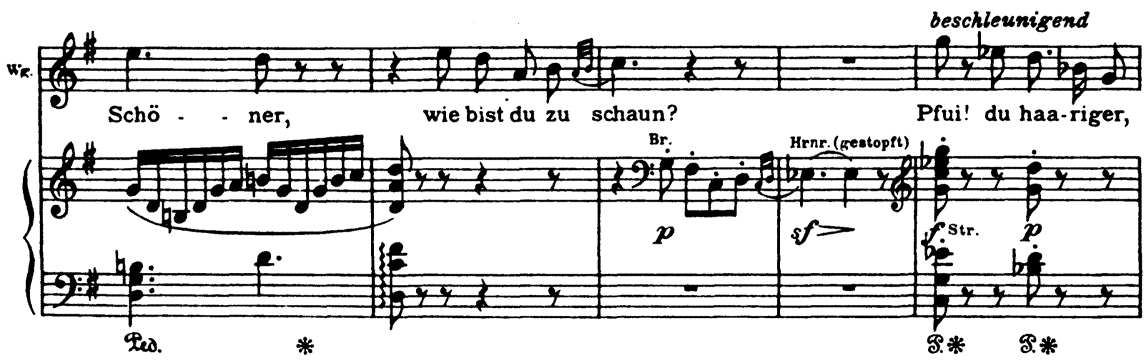

Figure 7. : O.R., I, 22

sur le 'wie bist du zu schaun ?',13 il faut reporter sur la voix de Woglinde les pizzicati des altos qui suivent. Les seules indications spécifiées par Wagner visent à éviter les effets normaux : il écrira piano dans une situation où on est enclin à chanter mezzo forte.

J.J.N. : Donc, pour contrer la tendance naturelle du chanteur?

J.T. : Exactement. On voit bien que les indications de Mottl ${ }^{14}$ concernent surtout des cas particuliers qui ont dû se produire au moment des répétitions de 1876 . Il y a beaucoup de choses qui dépendent des individualités. Pour tel chanteur particulier, à tel moment, ce sera mieux qu'il chante mezzo forte ou piano alors qu'il vaudrait mieux que tel autre chante forte. Cela dépend du caractère de la voix. Et comme notre objectif, vous le savez, était d'obtenir un texte particulièrement compréhensible, on a choisi pour chacun les moyens qui permettent avant tout la clarté de la diction.

13. 'Quel est ton aspect ?'

14 Il s'agit des remarques de Wagner, lors des répétitions du Ring en 1876, consignées par son assistant Felix Mottl. 
J.J.N. : Donc, l'absence de précisions, dans la partition vocale, vous laisse le maximum de liberté ?

J.T. : Oui. Il arrive que j'en profite pour créer un contraste entre l'orchestre et la voix. De façon générale, j'ai recherché une énonciation simple, qui tourne le dos au pathos et qui se mette au service du drame.

J.J.N. : Le ton d'une conversation?

J.T. : Oui, dès le début, avec les filles du Rhin. On obtient le naturel et la simplicité en respectant des petits détails. Dans le 'wachst du allein', 15 il suffit d'abréger la voyelle $a$ de 'wach', d'insister sur les deux $l$ de 'allein' avec un petit coup de glotte. Même chose immédiatement après : 'Mit Wellgunde wär ich zu zwei'16 Il faut souligner le mot 'zwei'; c'est ça qui est important, ici. Le $i$ de 'sicher' doit être aussi court que le $a$ de 'wachst'. Dans tous les cas, il faut insister sur le $w$ de 'wach' : on sait combien il serait important pour tout le monde que les filles veillent sur l'or soigneusement.

J.J.N. : Il y a, dans cette première scène, des moments que les metteurs en scène et les chefs d'orchestre ont naturellement tendance à 'solenniser'.

J.T. : Oui, au moment de l'apparition de l'or, puis du renoncement à l'amour. Pour l'apparition de l'or, on a évité de faire quelque chose de traditionnel. Les filles, certes, sont impressionnées, mais nous avons surtout voulu prendre l'or au premier degré, non pas comme un symbole, mais comme quelque chose qui, littéralement, excite les filles.

15 'Veilles-tu seule ?'

16 'Avec Wellgunde, nous serions deux !' 

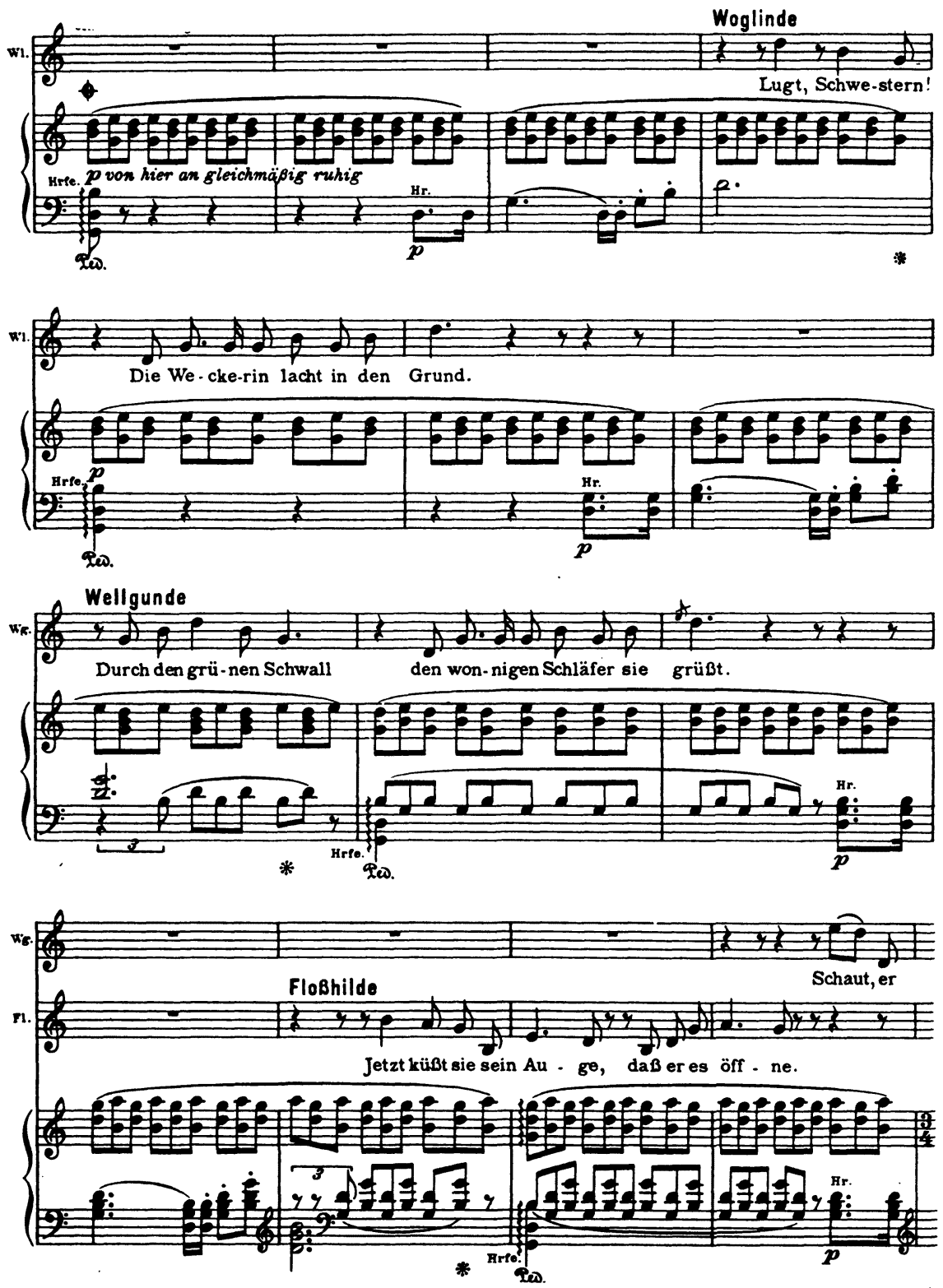

Figure 8. : O.R., I, 36-37 
'Lugt, Schwestern!/Die Weckerin lacht in den Grund'17 : il y a le $u$ de 'lugt' qui est relativement court, et surtout le rythme - celui de 'Weckerin' en particulier - qui traduisent cette excitation. On peut se permettre d'insister sur les consonnes : 'Durch den grünen Schwall/den wonnigen Schläfer sie grüsst'18 Le $g r$ de 'grüsst' tombe sur une appoggiature et on note que le 'jetzt' commence après le deuxième temps : la syncope, elle aussi, traduit l'excitation.

Boulez était entièrement d'accord avec cette manière d'exploiter les ressources du texte, car il a horreur que ce moment ne devienne religieux. D'ailleurs, il tient à ce que l'orchestre conserve ici sa plus grande régularité et que les filles maintiennent le piano.

J.J.N. : On a souvent adopté un ton solennel, également, lorsque le thème du renoncement à l'amour apparaît pour la première fois :

17 'Voyez, soeurs ! La lueur qui éveille rit dans l'abîme !'

18 'A travers les flots verdâtres, / elle salue le ravissant dormeur.' 

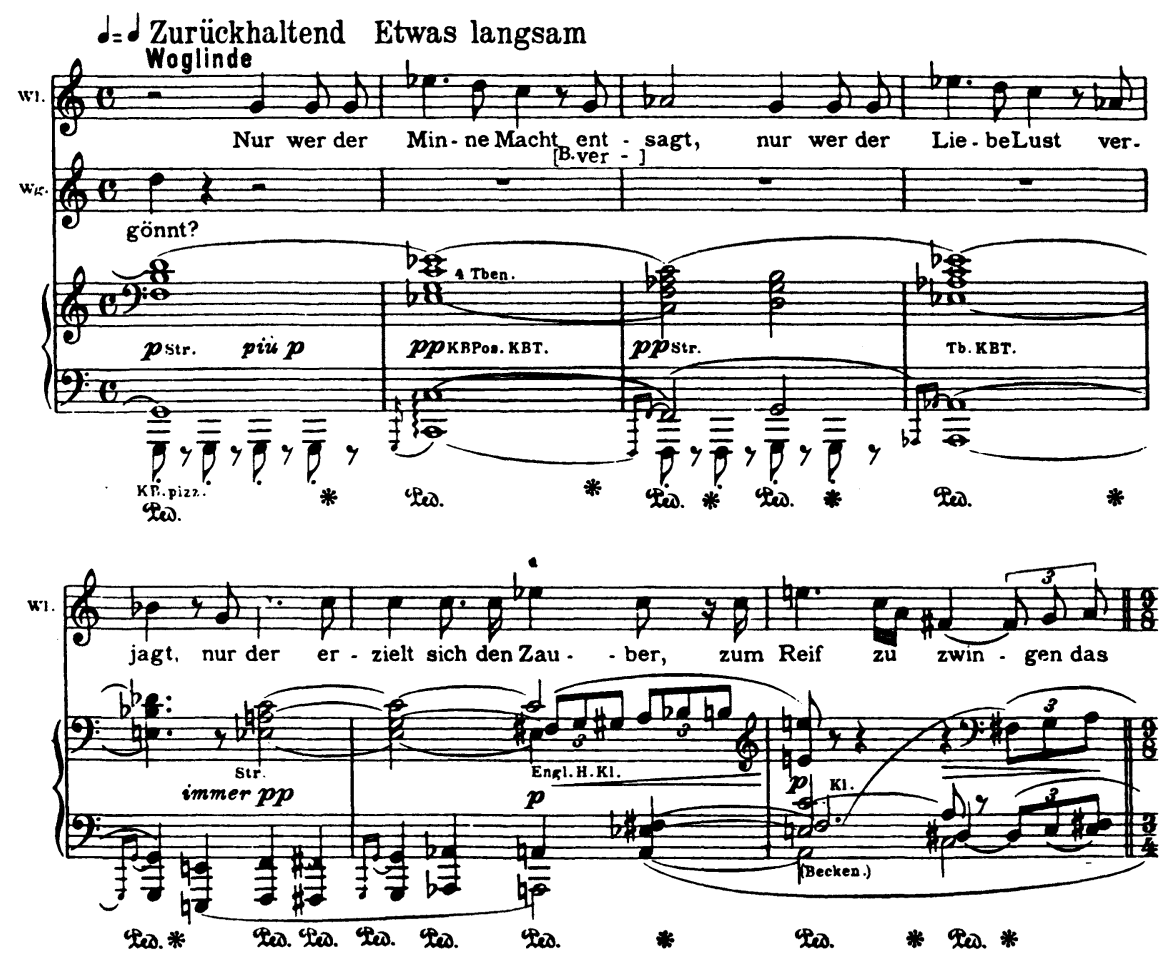

Figure 9. : O.R., I, 50-51

J.T. : Oui, et il n'y a vraiment rien dans la partition qui le demande. L'orchestre reste piano et même pianissimo. Il y a ces petites pauses avant 'versagt' et 'verjagt' qui traduisent une certaine malice : les filles ne croient pas, à ce moment, Alberich capable d'un tel renoncement, alors elles le provoquent...

J.J.N. : Pour Chéreau, seule la méchanceté des filles explique qu'il soit poussé à un tel geste ! On retrouve le ton naturel de conversation plus loin. 
J.T. : Tout d'abord, dans le dialogue Wotan-Fricka de la scène suivante. Le problème, là, c'est que les artistes chantaient trop et ne parlaient pas assez. Wagner - Mottl l'a noté - parle de 'scènes de dialogue où le tempo doit couler' (O.R. II, 67). Je prends un exemple :

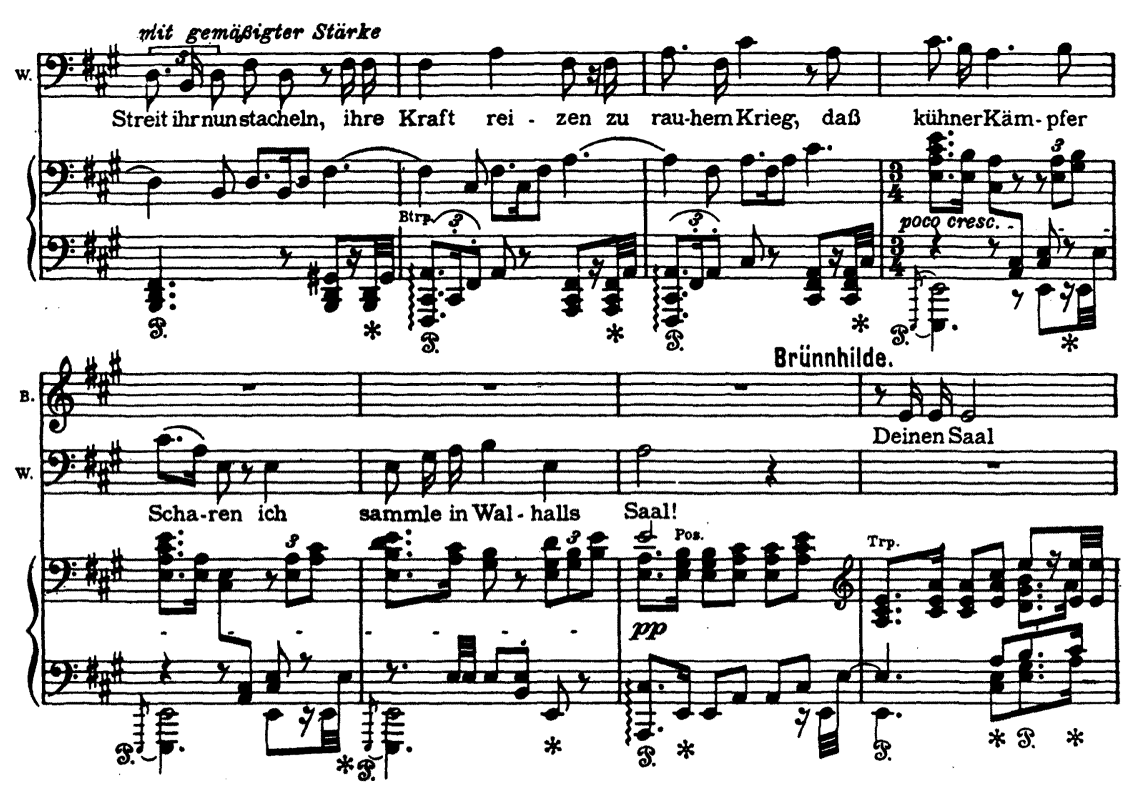

Figure 10. : O.R., II, 77

En fait, ils savent ce qu'est le Walhalla, ce n'est pas la peine d'insister. Il est beaucoup plus important, dramatiquement, qu'on respecte la progression relativement lente de cette scène, et si on donne une importance trop grande à ces deux mots-là, 'Walhalls Saal', on n'aura plus le moyen de graduer la montée de l'intensité. Chéreau tient à ce que Wotan trahisse, ici, une certaine amertume, avec quelque chose de sardonique dans la voix. Mais tout ça reste muet. Brünnhilde cherche 
les raisons de ce qu'il dit, elle cherche une logique. Il y a beaucoup d'attente dans cette conversation.

J.J.N. : Vous parlez beaucoup de dialogue et de conversation. Je suis tenté, comme je l'ai fait avec Pierre Boulez, 19 de vous demander si tout cela a quelque chose à voir avec le Sprechgesang.

J.T. : Mon sentiment, c'est que ça vient plus de Chéreau que de Boulez...

J.J.N. : Vous ne vous trompez pas !

J.T. : C'est drôle à dire, mais il est parfois nécessaire de trouver des formes d'expression qui soient loin de la musique ! Et ça vient davantage de la partition que de nous. Prenez le rôle d'Alberich :

19 De façon générale, Boulez n'aime pas le Sprechgesang schoenbergien et comprend mal la solution hybride du Pierrot lunaire. Si des hauteurs sont indiquées, il faut les jouer de façon précise. A plus forte raison dans le Ring. 

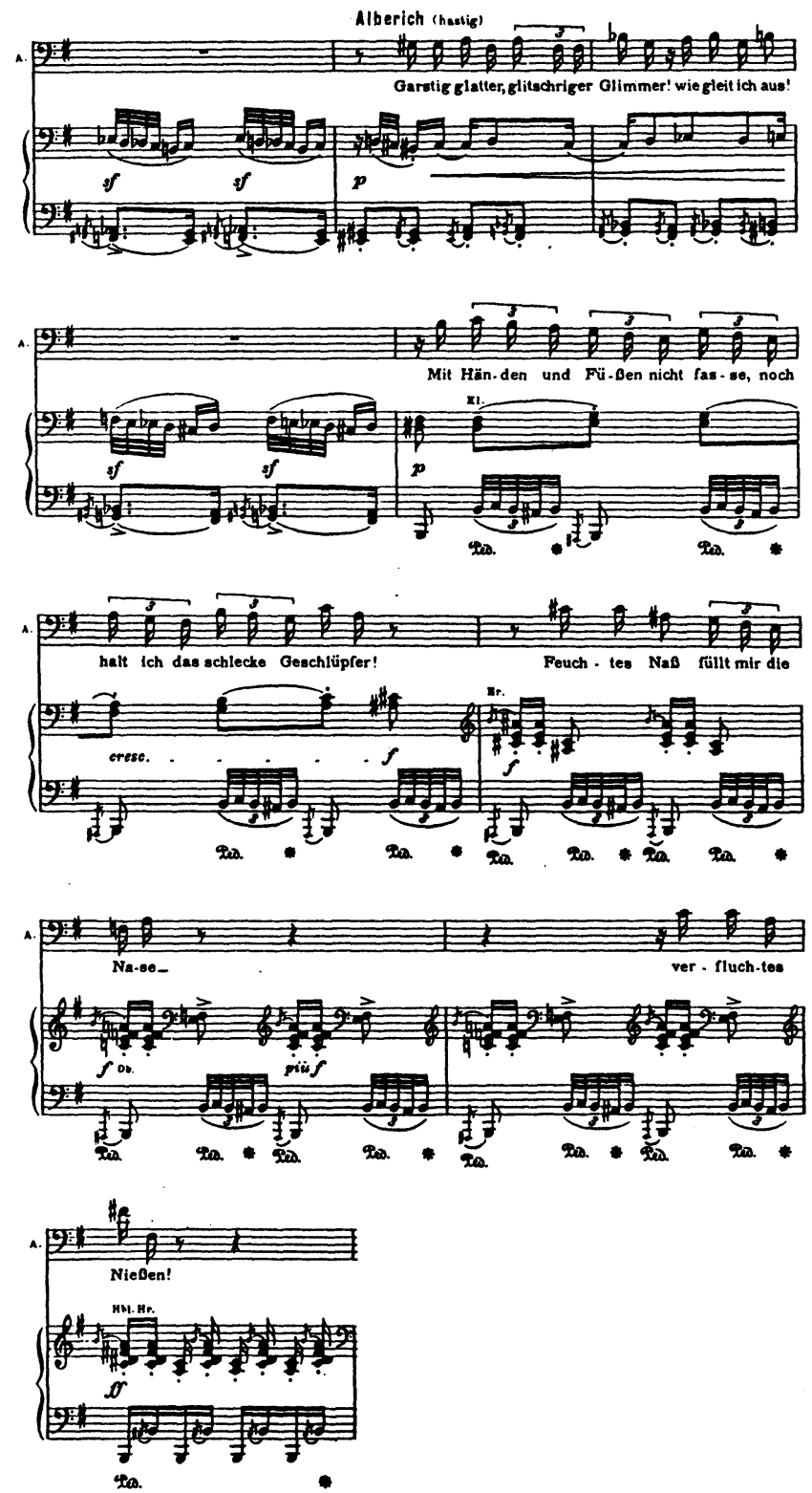

Figure 11. : O.R., I, 17-18 
On ne peut pas vraiment chanter chaque note. Il y a même en Allemagne une certaine tradition qui consiste à ne pas respecter ces triolets à la lettre. Je me demande si, à l'inverse, ce n'est pas cette façon de chanter Wagner qui a influencé Mahler et Schoenberg. Boulez voudrait qu'on soit plus près des notes, même avec l'intensité du jeu demandée par Chéreau. Il m'a fallu arbitrer ! Je ne crois pas ici qu'on puisse avoir à la fois l'expression ('hastig' - précipité), le texte dans le tempo et les notes. Or l'essentiel, dans notre conception du Ring, c'est l'efficacité dramatique : on ne peut pas comprendre les mots 'verfluchtes Niessen'20 si on chante ce qui est écrit. Il faut crier sur le nie ! Je ne suis même pas sûr qu'on arriverait à respecter les hauteurs en studio ou avec trois mois de travail supplémentaire.

Au début de $L^{\prime} O r d u$ Rhin, il me parait plus important, sur 'Pfui! der Garstige!', d'avoir une véritable explosion sur le $g$ plutôt qu'un sol et un si b musicalement 'jolis' :

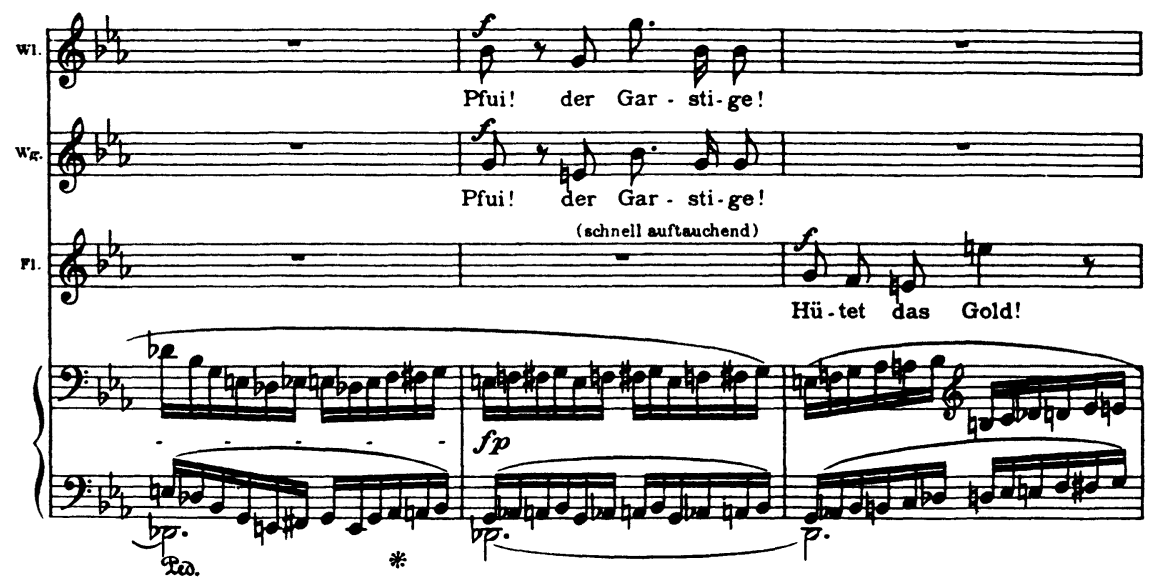

Figure 12. : O.R., I, 13 
C'est la couleur, ici, qui compte, la couleur au service de l'expression dramatique.

J.J.N. : Le mot 'Sprechgesang' n'est donc pas le plus approprié, ici ?

J.T. : Effectivement, il vaut mieux parler de 'récitatif', comme chez Mozart et Da Ponte. C'est presque parlé, mais il y a tout de même les rythmes et les notes ! Ce que je trouve fascinant, c'est la difficulté à rendre musicalement les rythmes de la langue allemande. J'en ai souvent parlé, en particulier avec Zednik : il considère qu'il est difficile de respecter exactement les rythmes de Wagner sans faire une injustice aux mots. Boulez, lui, réagit en compositeur : il trouve intéressant qu'on ne puisse vraiment déterminer le rythme précis des mots. Si on veut trouver la véritable expression, il faut accepter un certain rubato rythmique.

J.J.N. : Alors, vous, que faites-vous?

J.T. : J'essaie d'être toujours aussi près que possible du rythme de Wagner, mais sans perdre l'expression. Prenez l'entrée d'Alberich : 


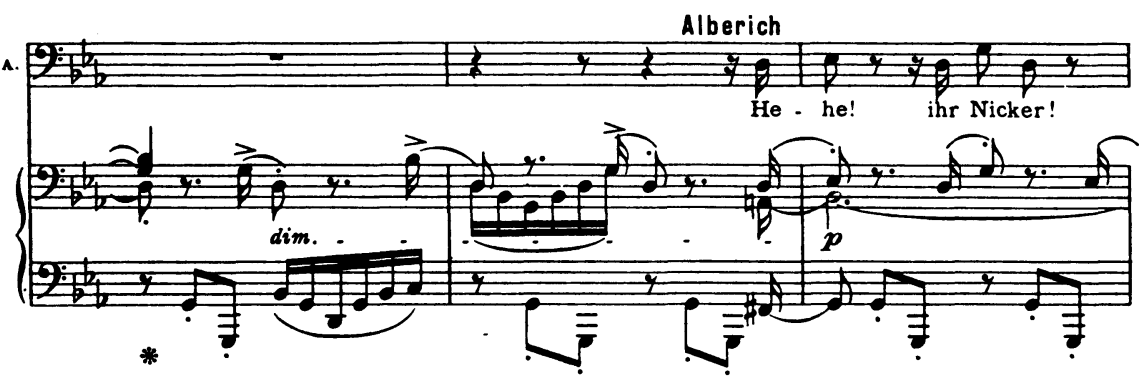

(Die Mädchen halten, sobald sie Alberichs Stimme hören, mit dem Spiele ein.)

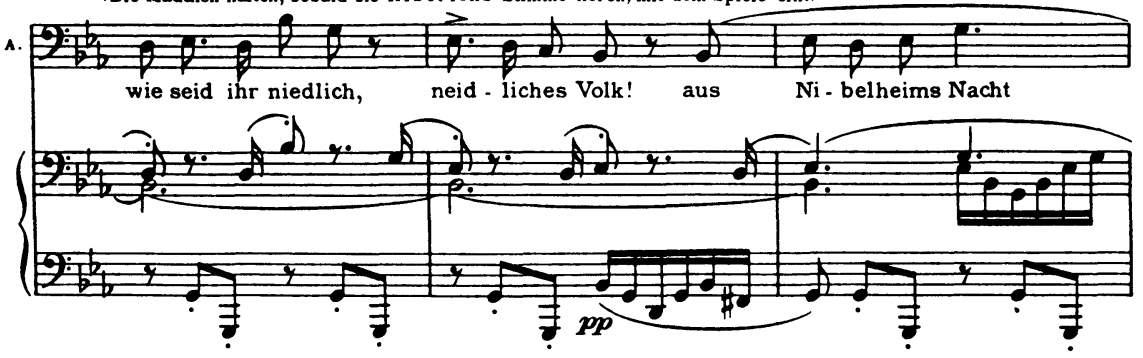

A.

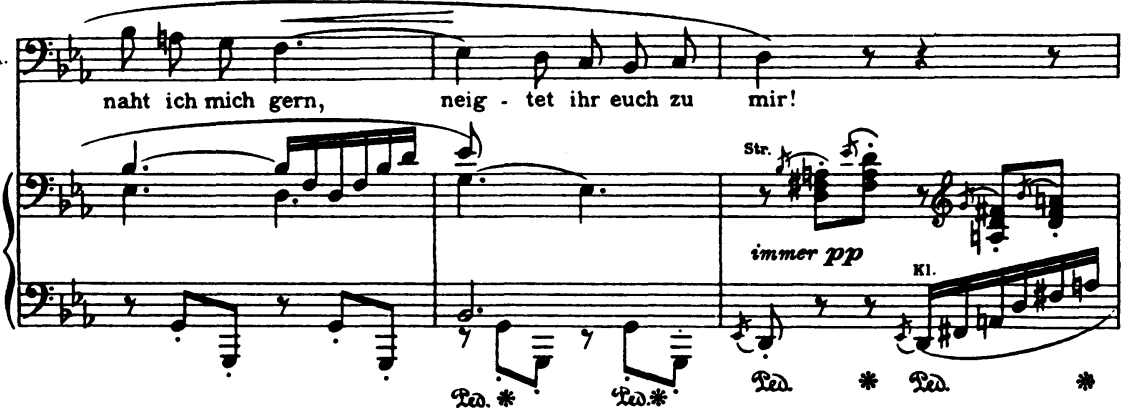

Figure 13. : O.R., I, 12-13

Le rythme est difficile, et on a, de plus, un legato explicite sur 'Aus Nibelheims Nacht/naht ich mich gern'21 Pourquoi ? Parce que

21 'Venant de la nuit de Niebelheim, / je vous approcherais volontiers.' 
Alberich désire les filles : c'est, chez lui, un sentiment vrai. Et il faut réussir à concilier l'expression de ce désir, le legato et la précision rythmique. Ici, ça doit chanter !

Vous trouvez le même genre de problèmes dans le rôle de Loge.
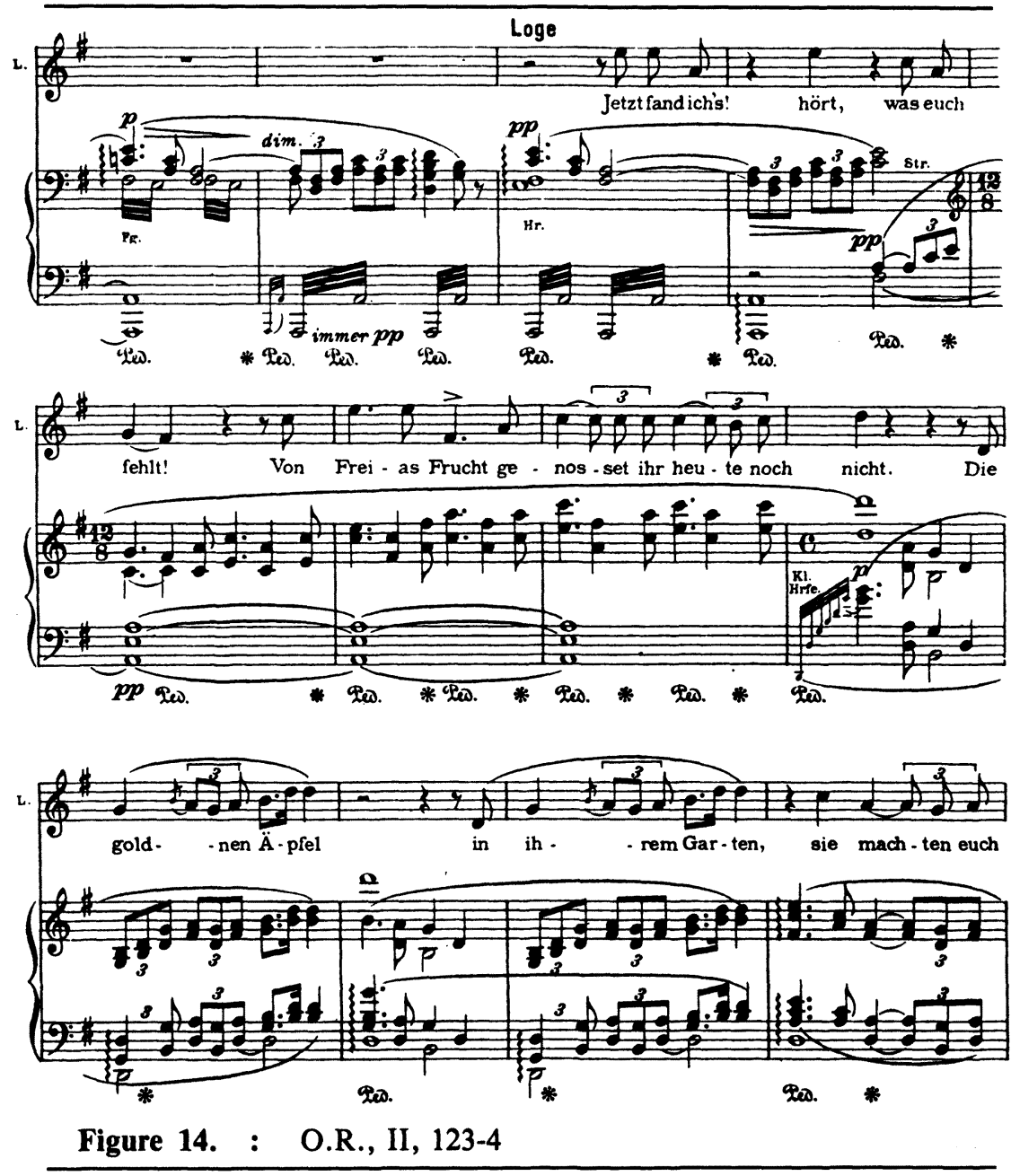
'Jetzt fand ich' !'22 : ce n'est ni parlé, ni chanté, c'est vraiment du récitatif. Dans le 'hört, was euch fehlt', ${ }^{23}$ il faut insister sur la dernière voyelle. Pour moi, les legati qui suivent ('Die goldnen Äpfel...') indiquent l'ironie : on doit exprimer la sympathie, mais c'est une sympathie dérisoire, et Zednik a trouvé ici les couleurs qu'il faut pour rendre l'ambiguïté de l'attitude.

L'unité de notre Ring, c'est le drame. Pour nous tous, c'est lui qui domine. Si on veut rendre l'attente anxieuse de Gudrune, au dernier acte du Crépuscule, il faut réaliser un rubato qui n'est impliqué que par le sens des mots (C.D., III, 332-4). On ne peut pas jouer ça de manière carrée. Boulez et moi, nous étions convaincus qu'il fallait que cette scène soit presque soufflée, le plus piano possible, avec un mélange de passivité et de nervosité. 'War das sein Horn?'24 : assez libre. Le 'Nein!' : comme chuchoté. 'Lachen Brünnhildes/weckte mich auf'25 : legato et diminuendo jusque sur le auf qui doit être prononcé comme une manifestation de douleur. 'Ich fürchte Brünnhild'!'26 : a tempo et chuchoté. On peut exploiter ici le contraste avec l'orchestre, car Gudrune déteste Brünnhilde en même temps. 'Brünnhild'! Brünnhild'!/bist du wach?'27 : très retenu, avec un point d'orgue en arrière : avant - qui n'est pas écrit - et un autre après. Elle, Brünnhilde, était là avant moi. Et le nouveau 'War das sein Horn', très vite, presque sans tempo : il n'y a rien qui se passe, c'est l'attente nerveuse, il n'y a presque plus de musique, il n'y a plus que le drame.

\section{J.J.N. : Il y a énormément d'intensité dans tout ce passage.}

22 'A présent, j'ai compris.'

23 'Sachez ce qui vous manque.'

24 'Etait-ce son cor ?...'

25 'Le rire de Brünnhilde / m'a réveillée.'

26 'J'ai peur de Brünnhilde.'

27 'Brünnhilde ! ... Brünnhilde ! ... / Veilles-tu ?' 
J.T. : Oui, et pour rendre l'intensité, à notre avis, il faut faire tout le contraire du pathos. La vraie intensité chez Wagner, c'est le moment où l'on rentre en soi-même, où il y a le plus grand calme, et je voudrais vous en donner quelques exemples. 'Wie im Traum ich ihn trug,/wie mein Wille ihn wies', ${ }^{28}$ dit Wotan :

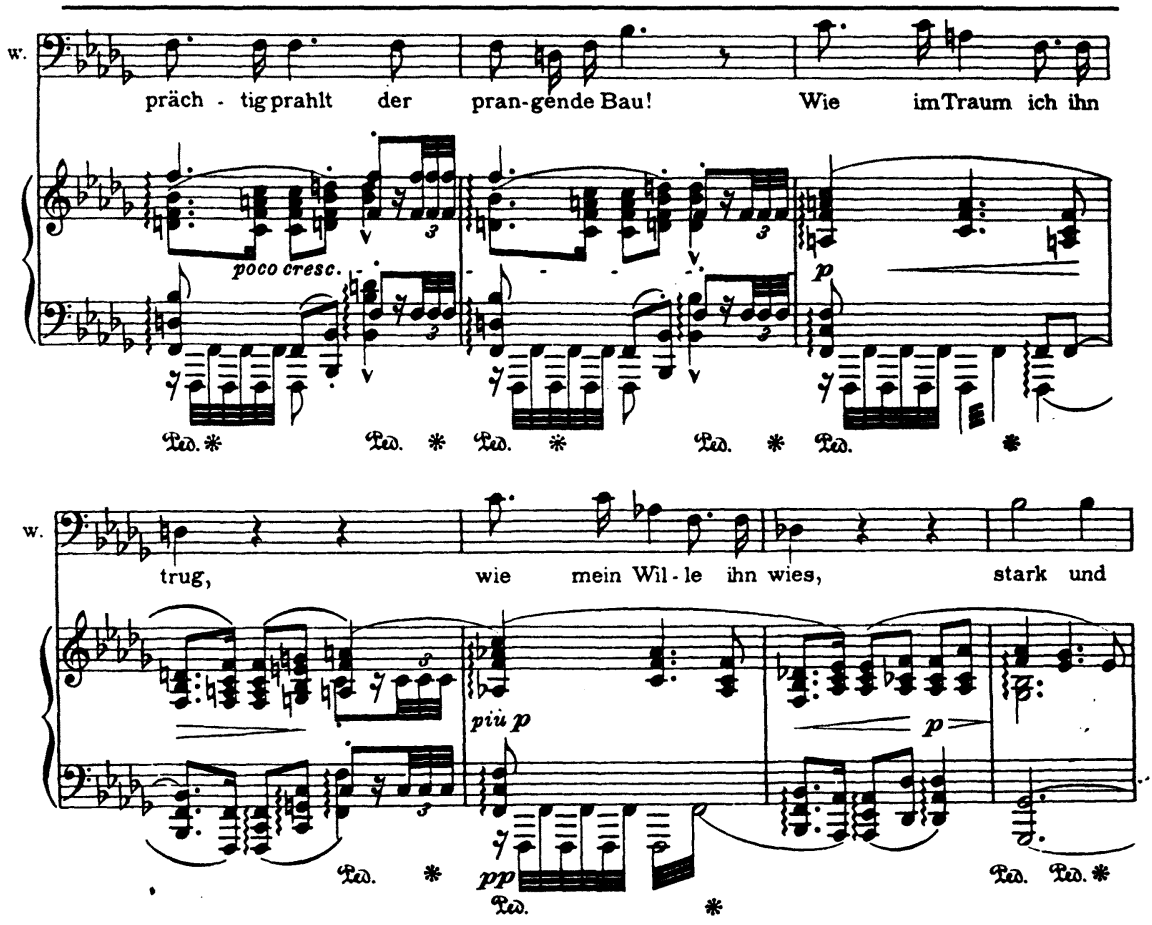

Figure 15. : O.R., II, 66-67

On pourrait s'attendre à mettre de l'emphase sur le mot 'Wille'. Mais c'est tout le contraire qui se passe : Wagner écrit più piano. C'est par le piano qu'il obtient l'intensification.

28 'Comme je l'ai porté en rêve, / comme mon vouloir (Wille) l'a fait voir'. 
Voyez cet autre moment décisif : 'Den Ring muss ich haben'29 :
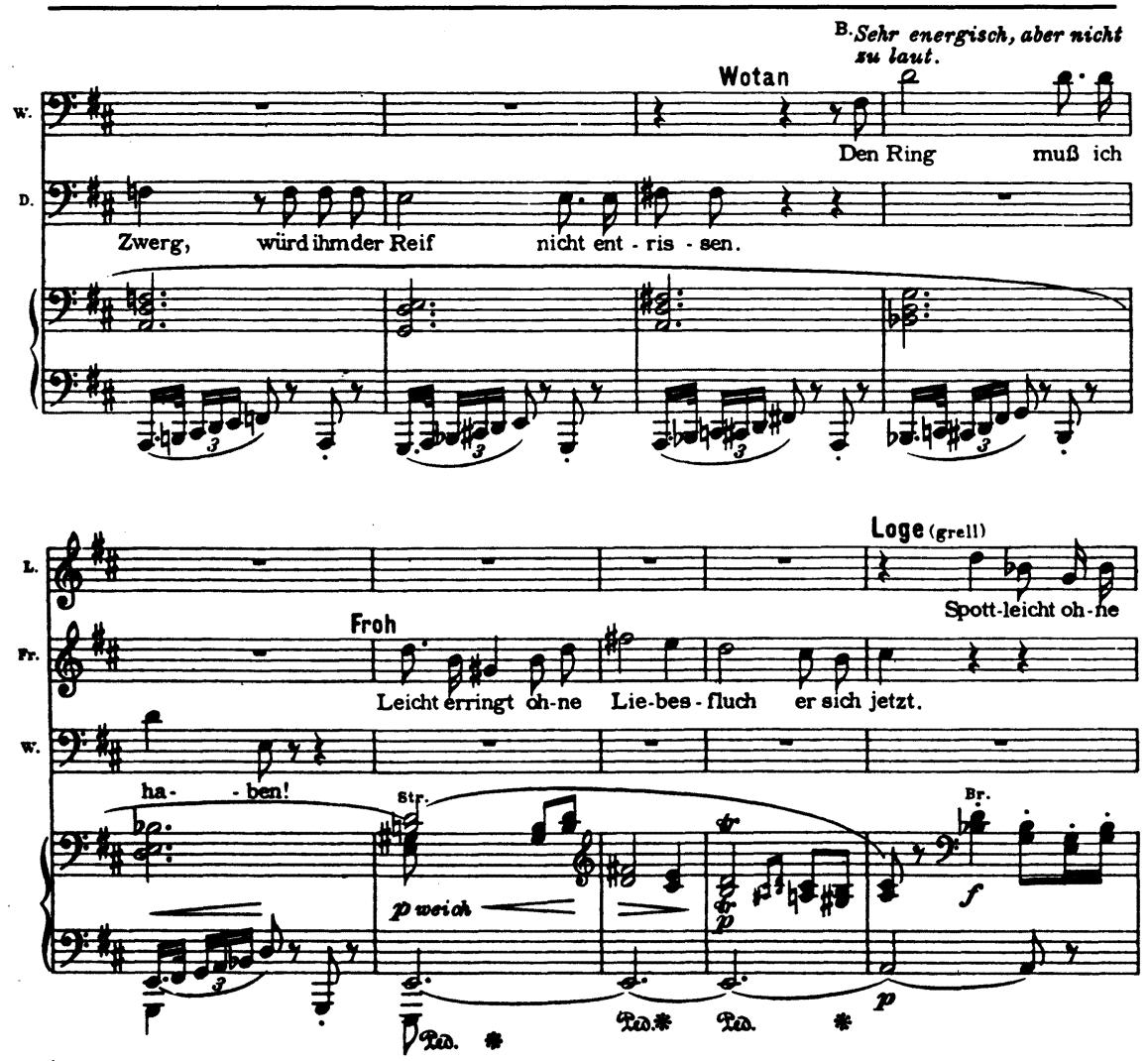

Figure 16. : O.R., II, 111-112

La partition indique que l'orchestre est toujours piano. Mottl a dû noter : 'Très énergique mais pas trop fort'. Evidemment : c'est une décision qu'il prend vis-à-vis de lui-même ! On peut multiplier les

29 'L'anneau, je dois l'avoir.' 
exemples : 'Sahst du nicht Loge?', 30 demande encore Wotan quand il l'attend anxieusement (O.R., II, 75). C'est plus intense que le 'Lass ihn drohn!'31 qui précède, donc je le demande plus piano.

J.J.N. : Pour ce genre de choses, encore une fois, il suffit de savoir lire!

J.T. : Oh ! je n’ai rien inventé :

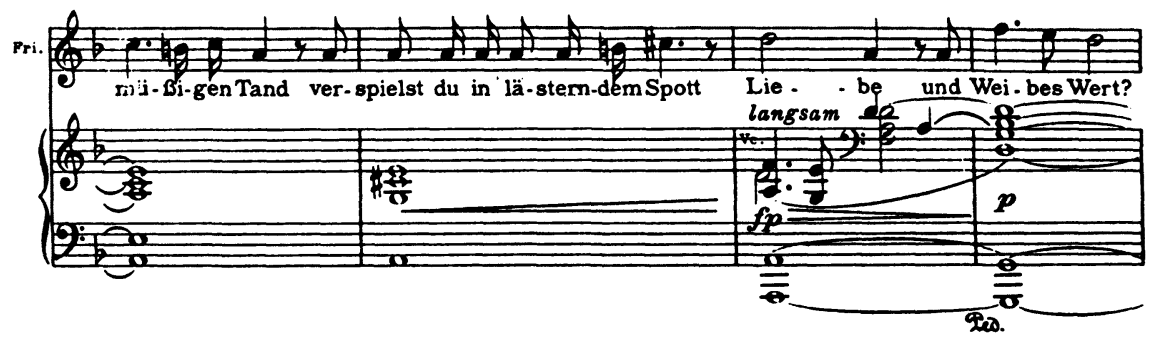

Figure 17. : O.R., II, 73

Pourquoi est-ce que Wagner écrit forte piano sur 'Liebe' et piano sur 'Weibes Wert'32 ? Parce que ce sont ces derniers mots qui sont les plus importants.

Une fois qu'on a compris le principe, ça permet de trouver la solution de problèmes particuliers. Ce passage m'embarrassait :

30 'N'as-tu pas vu Loge ?'

31 'Laisse-le menacer !'

32 'Le trésor de l'amour et de la femme.' 

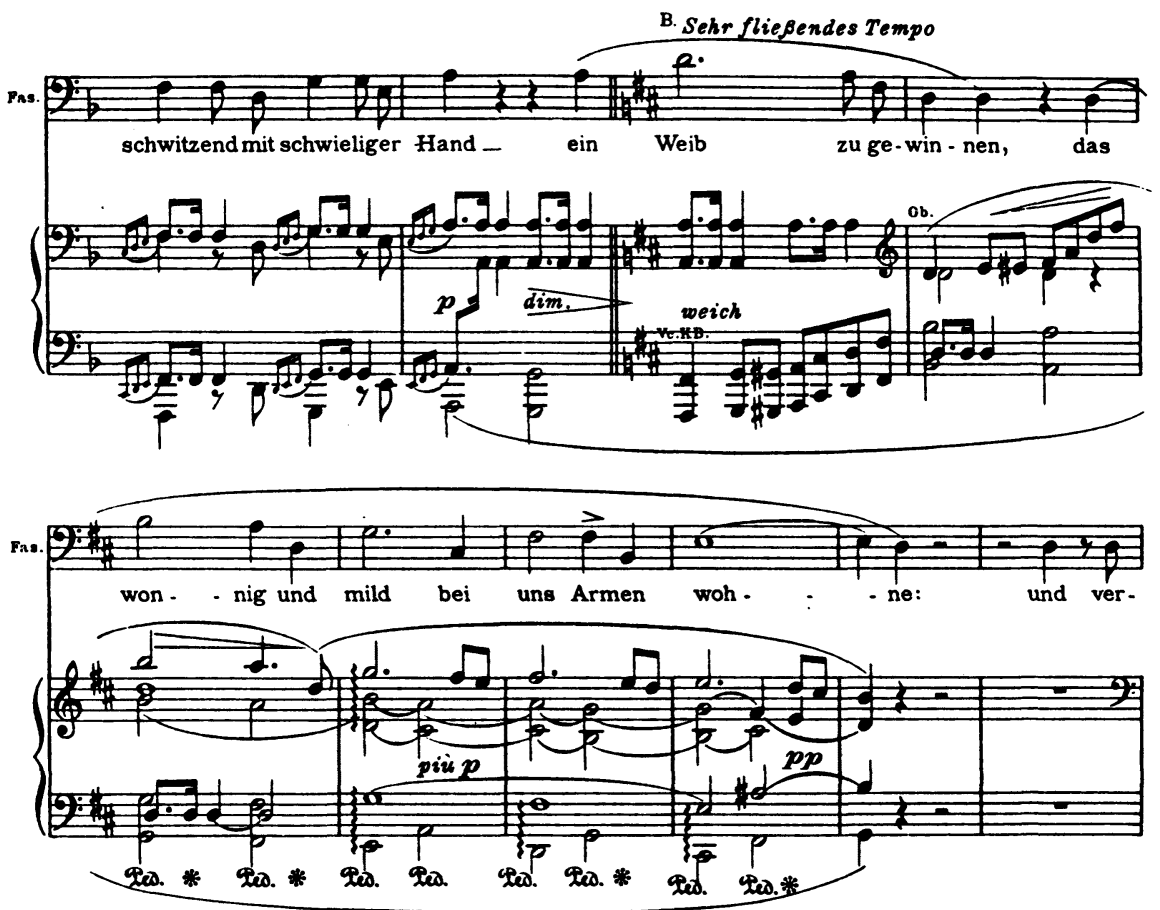

Figure 18. : O.R., II, 85

Wagner a précisé qu'il voulait un accent sur le mot 'Armen', qui est effectivement important du point de vue psychologique : en comparaison de Freia, nous sommes vraiment les pauvres, dit Fasolt, et il est absolument sincère. La difficulté, c'est que Fasolt est amoureux et nous avons un legato dont il ne faut pas perturber la grande ligne.

\section{J.J.N. : Qu'avez-vous fait ?}

J.T. : Pour ne pas rompre cette continuité, j'ai proposé une sorte d'accent à l'envers avec un coup de glotte et le mot 'Armen' plus piano que le reste. Ainsi, on réussit tout de même à le mettre en relief.

J.J.N. : Le procédé, encore une fois, vise à obtenir le maximum de clarté. 
J.T. : Oui, cela a été ma préoccupation constante pendant ces cinq ans, et la clarté ne s'obtient pas nécessairement en chantant fort, mais en articulant le plus possible. Ca n'est pas pour rien que le poème de Wagner est rempli d'allitérations : Fasolt : 'Kampf kiesten wir nicht'33 (O.R., II, 89). Donner: 'Schon oft zahlt ich Riesen den Zoll'34 (ibidem). Il n'y a pas de signes de legato sur ces deux vers, mais on pourrait concevoir d'en placer un. J'ai demandé à ce qu'on n'en mette pas, de façon à insister sur les sonorités et souligner chacun des mots. La couleur est souvent dans les consonnes, et spontanément, les chanteurs n'aiment pas insister sur elles. $\mathrm{Ca}$ ne vient pas naturellement. Certes, il y a de grands moments de legato dans ce Ring (le duo Siegfried-Brünnhilde, la fin du Crépuscule) mais dans $L$ 'Or $d u$ Rhin (sauf la scène d'Erda) le premier acte de la Walkyrie, et presque tout le second acte, il faut que la voix soit le véhicule des mots.

J.J.N. : Est-ce qu'il n'y a pas eu plus d'un problème avec le tempo ?

J.T. : Oui. Dans le vers 'Was keinem in Worten ich Künde', ${ }^{35}$ le Stabreim de Wagner, ici, souligne à la fois le rythme de la mélodie et celui du sens :

33 'Ce n'est pas le combat que nous avons choisi.'

34 'Souvent déjà j’ai payé / l'impôt aux Géants.'

35 'Que demeure donc à jamais/inexprimé/ce que je ne puis révéler à personne.' 

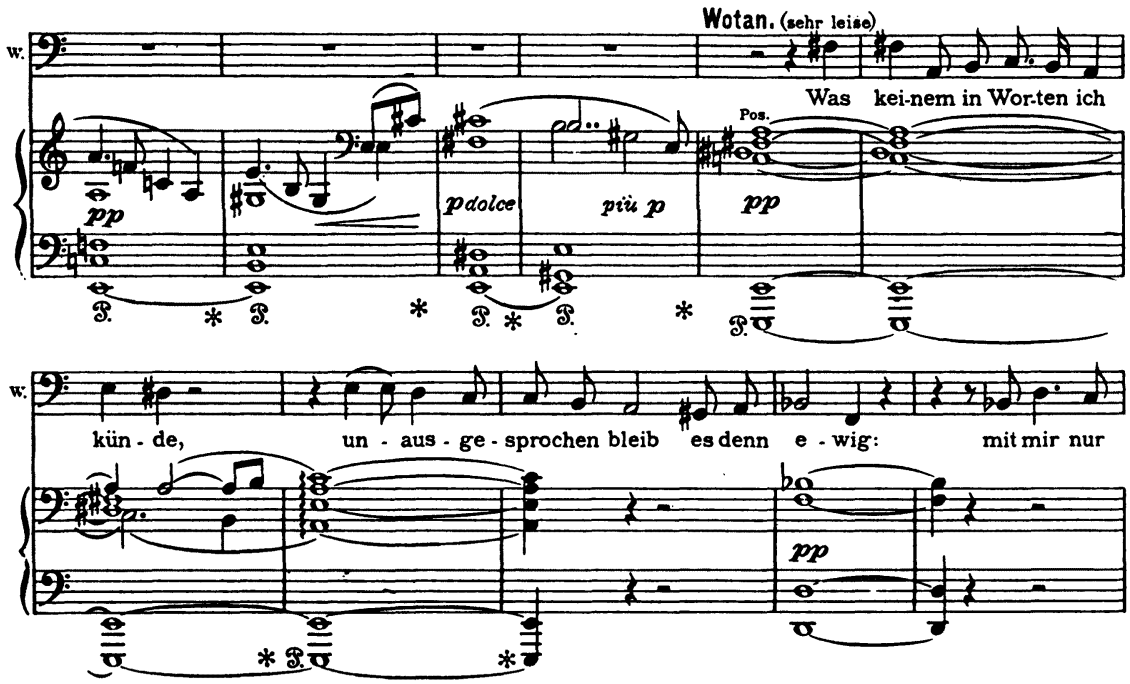

Figure 19. : W., II, 122

Je demande un coup de glotte sur ces consonnes, mais Boulez n'aime pas qu'on exagère l'attente provoquée par cet aspect de l'articulation. Pour respecter le tempo strict du chef, le chanteur doit donc s'ajuster.

J.J.N. : On a souvent critiqué la qualité du poème de Wagner précisément en raison de l'envahissement des allitérations. Cherchez-vous à les atténuer?

J.T. : Pas du tout. Les allitérations dérangent lorsqu'on lit le texte sans la musique, mais elles ont été conçues pour donner un côté musical et rythmique au texte. Il n'y a aucune raison de vouloir couvrir ce qui est évident. Au début de L'Or du Rhin, par exemple : 

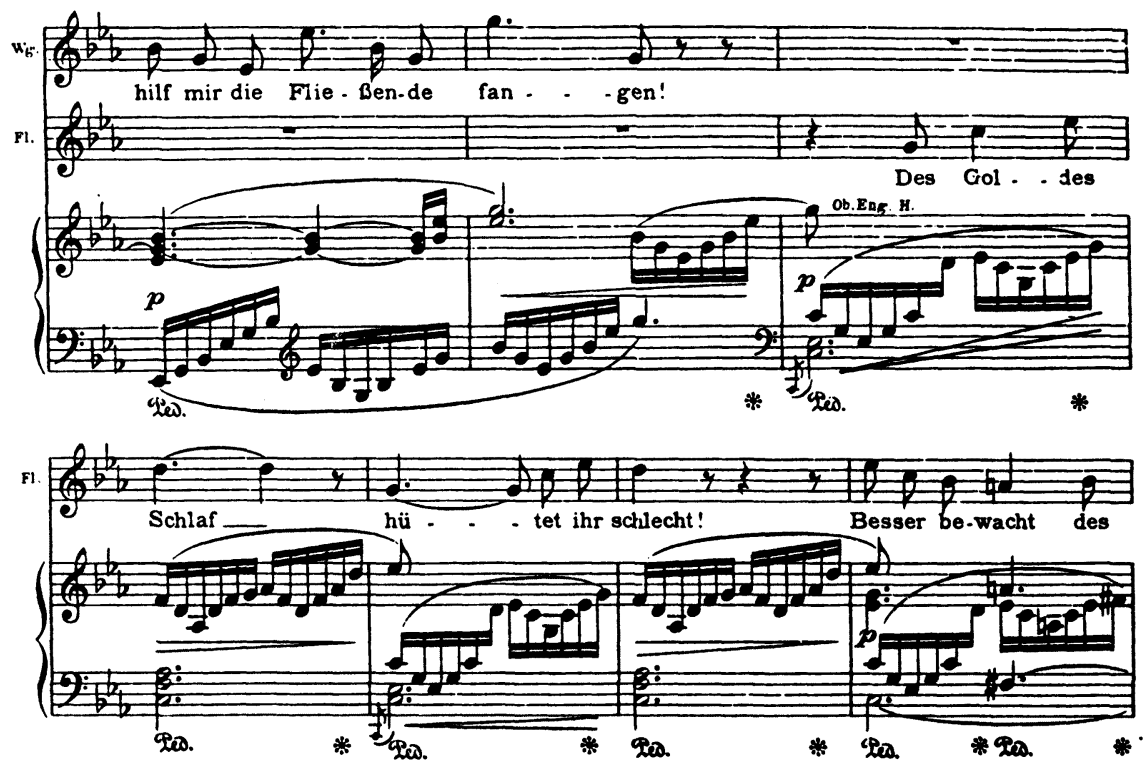

Figure 20. : O.R., I, 11

Les accents sur Schlaf et schlecht soulignent à la fois le parallélisme des sonorités et celui du rythme harmonique (I-II-I-II) : il faut respecter cette connexion entre l'écriture musicale et l'écriture du poème. Et je pourrais multiplier les exemples. ${ }^{36}$

J.J.N. : Vous venez de détailler les procédés. Il serait intéressant de ramasser tout cela à propos d'un passage significatif. Je pense au monologue de Wotan, dans le deuxième acte de la Walkyrie.

36 Dans le troisième acte du Crépuscule : 'Das Gold wiedergabe' (p. 265), 'ein goldner Ring glänzt' (p. 272), 'in fernster Zeit zu zeugen den Tod' (p. 282), 'so heissen wir's dir' (p. 283). 
J.T. : C'est un des cas où je suis parti d'une des trouvailles de Chéreau...

J.J.N. : ...qui est particulièrement réussie. ${ }^{37}$

J.T. : McIntyre-Wotan a le dos tourné au public et se parle dans un grand miroir. Son visage n'est éclairé que par un spot venu de la salle qui frappe le miroir et se réfléchit sur son visage. Pratiquement, il y avait deux problèmes : McIntyre ne peut plus suivre les battues de Boulez mais doit chanter a tempo; et la plupart des spectateurs ne voient pas les lèvres du chanteur.

On s'est vite aperçu qu'on obtenait l'efficacité dramatique la plus grande, correspondant à ce que voulait Chéreau, en maintenant un piano constant et en attachant la plus grande attention au texte. Boulez adopte un mouvement qui n'est pas exagérément lent. 'Mässig langsam' (modérément lent) dit la partition. Tout cela crée un moment d'intensité et d'intimité extraordinaire. Il n'y a plus rien. Le zéro du Ring. Wotan, pour la première fois de sa vie, voit la vérité en face.

Tout vient du rythme, et comme on ne voit plus les lèvres, il faut soigner particulièrement l'articulation des mots. La dynamique, ici, n'est pas vraiment importante, puisqu'il s'agit de maintenir le piano. McIntyre ajoute de toutes petites nuances, à l'image de ce petit détail précisé par Wagner sur 'fluchte' :

37 Cf. l'analyse détaillée de cette scène in Nattiez $1983:$ 139-144. 

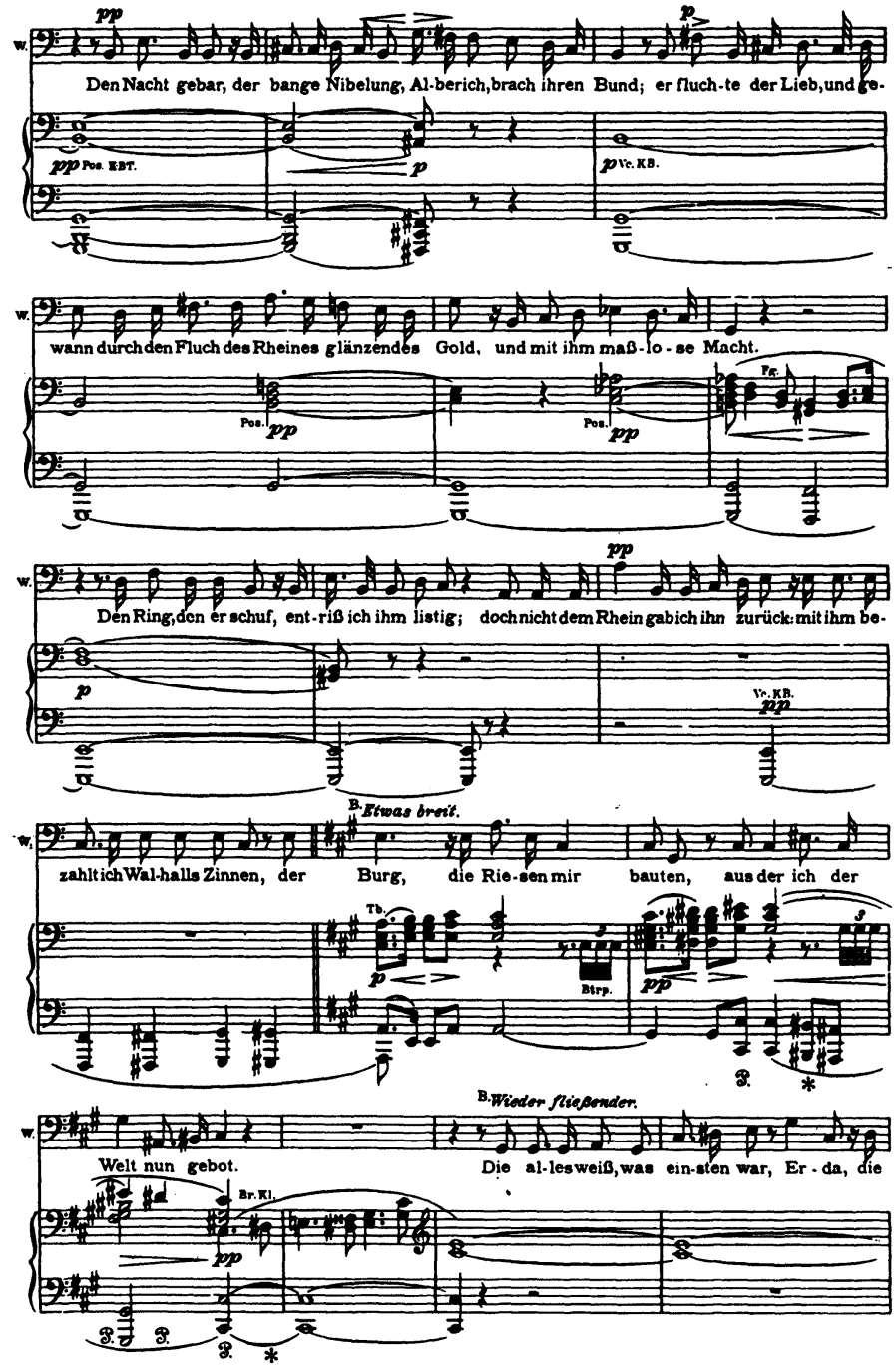

Figure 21. : W., II, 124

Ensuite, pour marquer la nostalgie de Wotan à l'égard du Rhin, il prononce Rheines avec le pianissimo indiqué à l'orchestre. Dans ' $m$ it ihm 
masslose Macht', ${ }^{38}$ ces $m$ sont importants : Wotan fait une espèce d'auto-critique. Je lui fais prolonger le $m$ de 'ihm' et le $a$ de 'mass'. Il y a de plus une question harmonique : la sonorité de Rheines évoque une pureté que souligne, au-dessous, l'accord majeur. Au contraire, l'accord mineur sous masslos sonne comme une appoggiature de tristesse et de menace. La différence de couleur entre les deux voyelles renforce l'intervalle de triton entre le la de 'Rheines' et le mi bémol de 'masslos'. Il y a là un mélange de peur et de pureté des sentiments.

J.J.N. : Et on rencontre un mélange analogue de sentiments juste après.

J.T. : Oui, quand il est question du Walhalla : 'der Burg, die Riesen mir bauten'. ${ }^{39} \mathrm{Ce}$ n'est pas parce que l'orchestre esquisse le thème du Walhalla qu'il faut se déchaîner. D'ailleurs, l'orchestre doit rester piano et pianissimo C'est à la fois fier et nostalgique. De la nostalgie, parce qu'il sent bien que cette époque heureuse, associée au château, va finir. Et pendant cette période, il a connu une expérience inoubliable avec Erda - dont il a eu les Walkyries. Elle l'impressionne toujours. Sur ce passage : 'Die alles weiss,/was einstens war,/Erda, etc.', 40 il chuchote. Avec le miroir, c'est encore plus facile. On a expérimenté le même passage en acoustique 'ouverte', avec Wotan face au public, mais ça ne marchait pas.

C'est tout cet ensemble de facteurs qui a contribué, je crois, au grand succès de cette scène auprès du public et de la critique...

J.J.N. : ... là où, habituellement, tout le monde s'ennuie si profondément! Avec sa méchanceté coutumière, Hanslick, rendant compte de la Walkyrie, écrivit que ce monologue avait été la grande surprise des plus acharnés des wagnériens : un moment de mortel ennui !

\footnotetext{
38 'par lui, puissance sans mesure'.

39 'le burg que les Géants m'ont édifié'.

40 'Celle qui sait tout / ce qui fut autre fois, / Erda...'
} 
Un peu plus loin, on sent bien que McIntyre, sur le mot 'blindem'41 suit le piano subito qui intervient à l'orchestre :
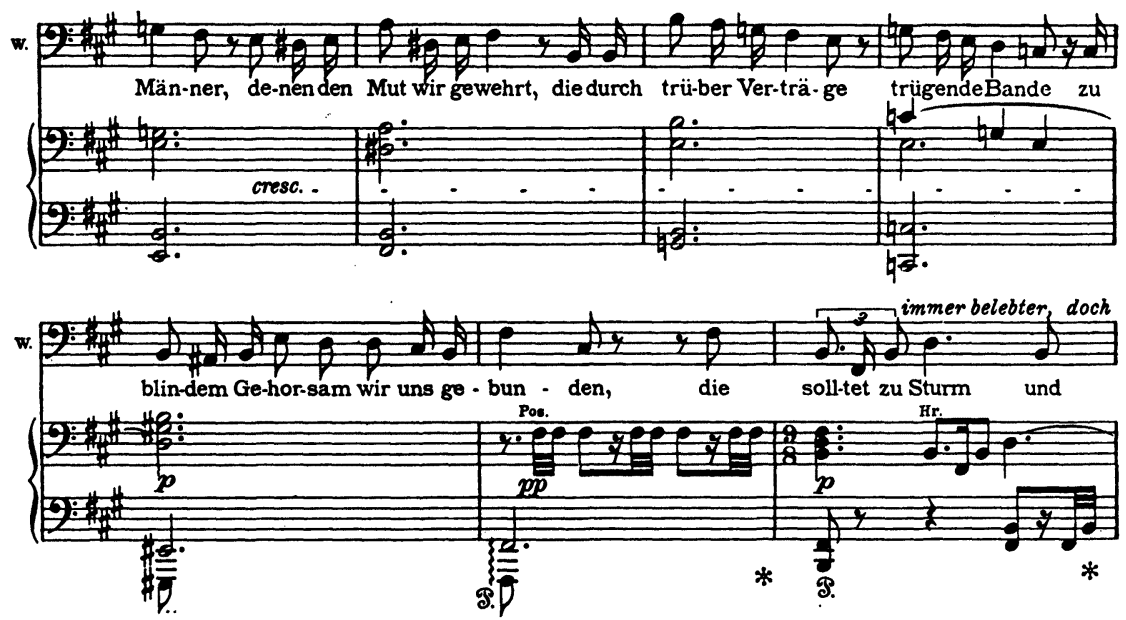

Figure 22. : W., II, 127

J.T. : Effectivement, à partir de ce moment-là, Wotan devient autocritique et auto-ironique.

J.J.N. : Tout ceci donne raison à Boulez, qui considère qu'il n'a pas besoin de regarder le texte pour s'adapter musicalement à la psychologie des personnages, parce que, de toutes façons, structures musicales et structures dramatiques se correspondent.

J.T. : Absolument ! Il suffit de respecter la partition. C'est curieux, parce que c'est un problème dont je n'ai jamais discuté avec lui. Sans doute est-ce parce que ça n'était pas nécessaire et que, fondamen-

41 'aveugle' 
talement, nous considérons tous les deux le respect des nuances comme un credo esthétique. C'est sans doute pour cela que, au niveau du résultat, nous parvenons à une parfaite coïncidence.

J.J.N. : Oui, mais en ce qui vous concerne, vous orientez tout de même l'interprétation musicale du texte en fonction, aussi, de ce que vous dicte le sens des mots et des situations, en particulier au plan psychologique.

J.T. : Sans aucun doute. Quand je demande à McIntyre d'accentuer le $L$ de 'Leute' dans 'Nennt, Leute, den Lohn'42 (O.R. II, 80) adressé aux géants, j'utilise la consonne pour indiquer le mépris de Wotan. A la page suivante, Chéreau fait faire de grands gestes aux géants sur les fortissimi. Puis, il y a un piano subito : Fasolt n'est plus sûr de rien, il devient plus nerveux et plus effrayé. On peut le traduire dans un tempo un peu plus rapide et un certain changement de dynamique.

42 'Dites, gens, le salaire'. 

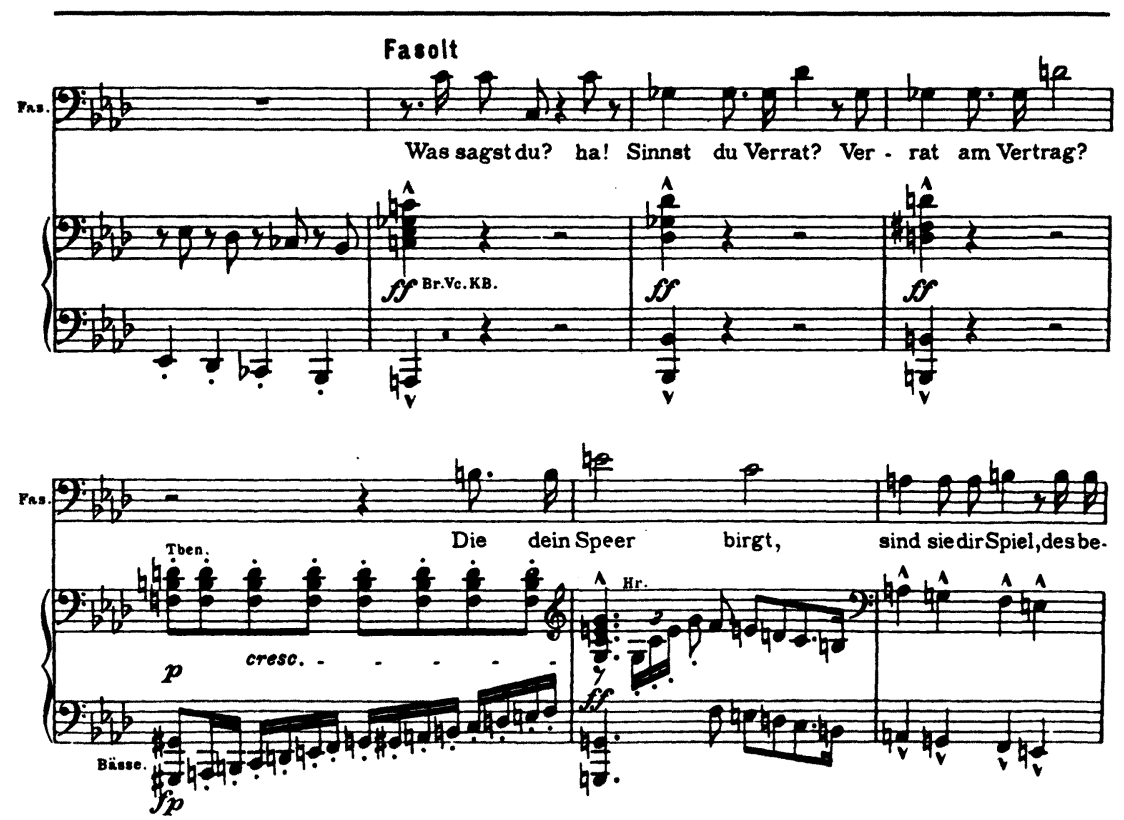

Figure 23. : O.R., II, 81

J.J.N. : Et en fonction de cette interprétation psychologique que vous faites du texte, vous proposez des dynamiques qui ne sont pas indiquées mais qui vous semblent justifiées.

J.T. : Oui. Par exemple, le cri de douleur de Freia : 

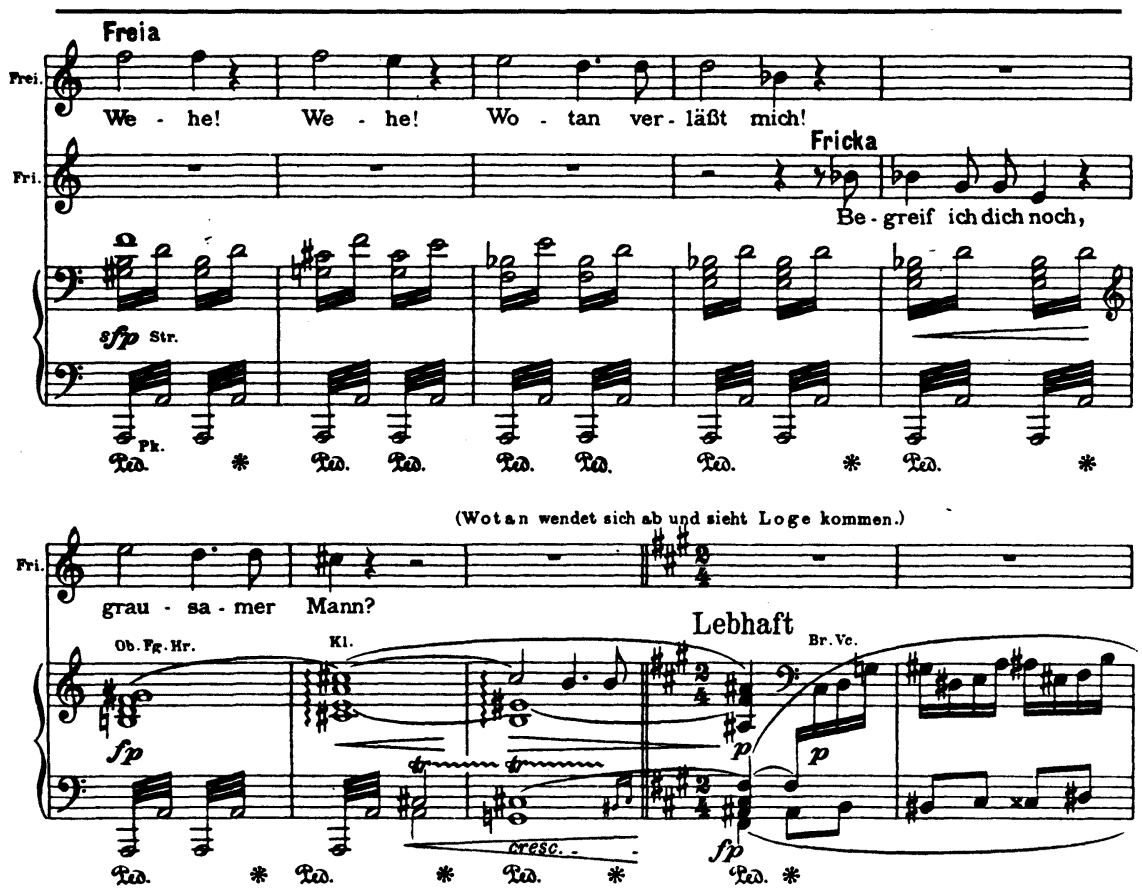

Figure 24. : O.R., II, 90

J'ai ajouté un decrescendo et un legato. A ce moment-là, Freia est un être sans activité, pauvre, passif. Elle comprend clairement que Wotan la laisse tomber et que les difficultés commencent. Elle ne doit donc pas crier, mais exprimer la douleur. Le contraste avec Fricka qui va être beaucoup plus dur et marcato n'en est que plus fort. C'est pourquoi je tiens au legato.

J.J.N. : Je constate que vous utilisez beaucoup l'exploitation des allitérations pour insister sur l'ironie.

J.T. : Oui, il y a beaucoup d'ironie dans cette oeuvre, surtout dans L'Or du Rhin. D'abord avec les filles du Rhin, qui ne cessent de se moquer d'Alberich. Au tout début, je voudrais que le ie de 'der Feind 
ist verliebt'43 (O.R. I, 15-16) soit autant piano subito que celui de l'orchestre. Avec la longueur de la voyelle, l'ironie s'installe, prolongée par les k de 'Der lüsterne $K a u z ' 44$ et de 'Lasst ihn uns kennen'. ${ }^{45}$

Plus loin (Ex. 25), lorsque Woglinde évoque le 'goldes Schmuck', ${ }^{46}$ je souhaite un $u$ assez court, et une résonance du $c k$ sur les cinq temps du $s i$, puis le $\ddot{a}$ de 'schmähte er nicht'47 très ouvert : elle se moque de lui, il ne comprend pas. Après quoi, elle lui emprunte son ton un peu plus rude et accentue le rythme de 'all seine Wunder'.48 Je l'ai déjà dit : l'or excite les filles.

43 'L'ennemi est amoureux'.

44 'Le drôle lubrique'.

45 'Faisons-nous connaître de lui'.

46 'L'éclat de l'or'.

47 'Il ne l'injurierait pas'.

48 '[s'il connaissait] tous ses prodiges !' 

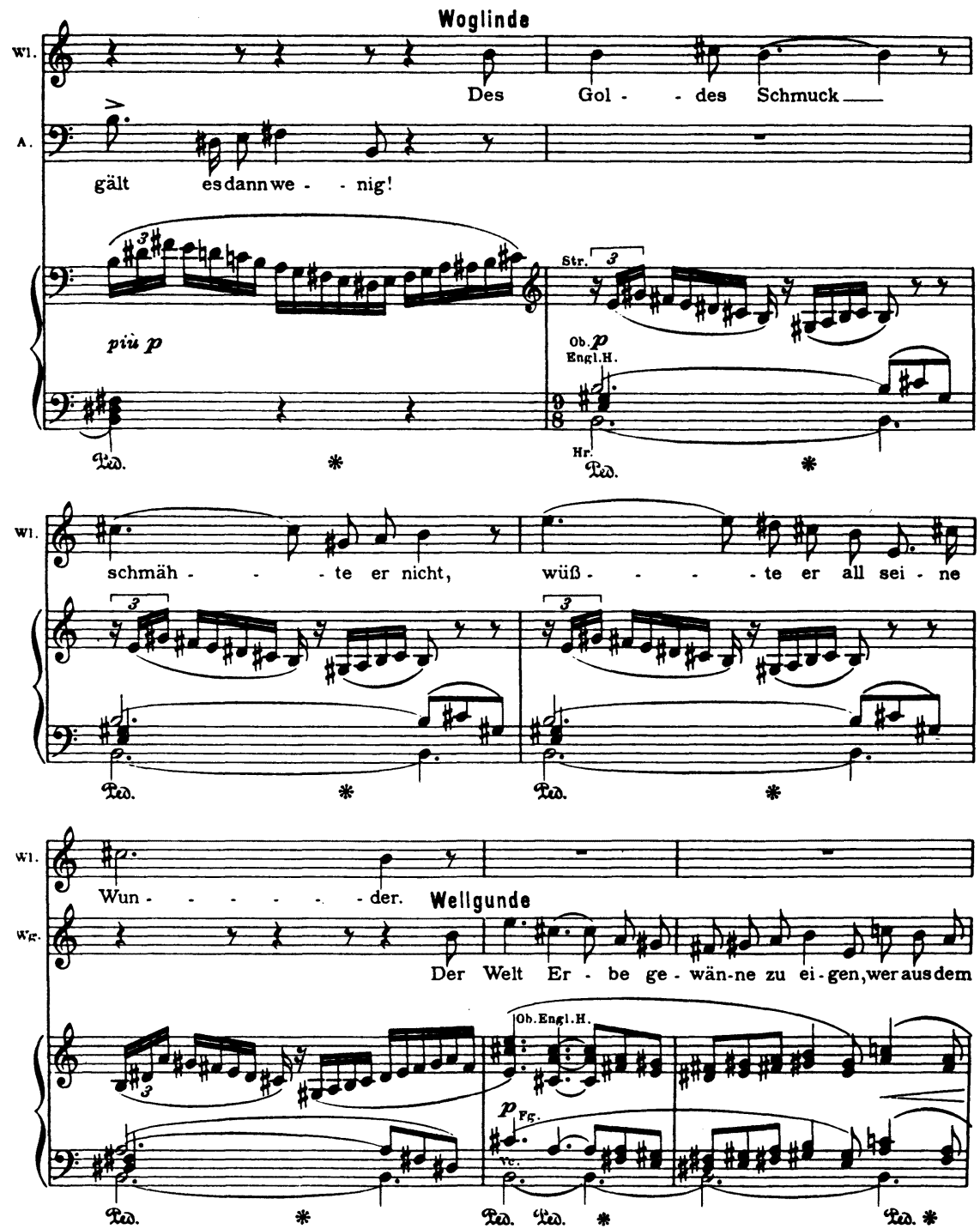

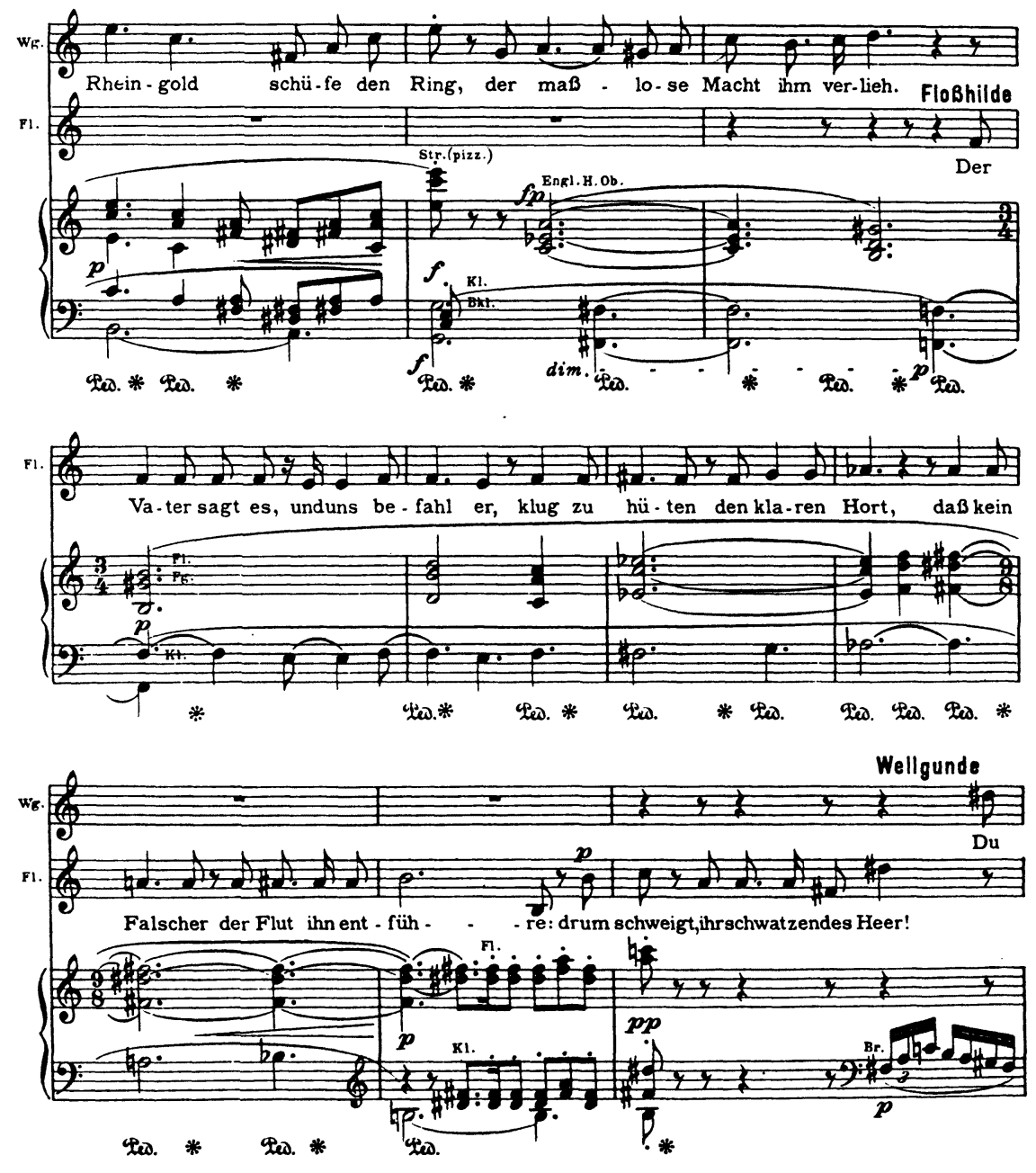

Figure 25. : O.R., I, 48-49-50 
Il y a là un élément de mystère qui n'a rien de mélodramatique. Wellgunde joue à tenter Alberich, elle ne croit pas que c'est très dangereux : voyex le piano subito de l'orchestre sur 'Rheingold', et surtout, le staccato très important sur le mot 'Ring'. Avec Flosshilde, c'est autre chose : quand elle parle de ce que son père a dit, on a le sentiment d'une sincérité certaine ; alors, j'ai demandé des couleurs de voyelles plus sombres. En même temps, il faut que ça reste piano, car, comme le remarque Chéreau, il faut toujours penser aux autres personnages présents sur la scène; or, Alberich ne doit pas entendre ce qu'elle dit.

J.J.N. : D’ailleurs, Wagner a précisé le piano sur la partie de Flosshilde lorsqu'elle demande à ses soeurs de parler moins fort! Ce qui me frappe, dans ce passage où les trois filles du Rhin se succèdent, c'est le soin que vous mettez à les distinguer.

J.T. : C'est quelque chose de capital dans notre Ring, et je crois qu'il faut parler de différenciation à tous les niveaux. D'abord, si on regarde le texte du poème et le texte musical de près, on constate que, pour un même personnage, ou dans un même passage, il y a beaucoup de différenciations à obtenir dans les couleurs et les dynamiques. Prenons ce passage de Wellgunde : 

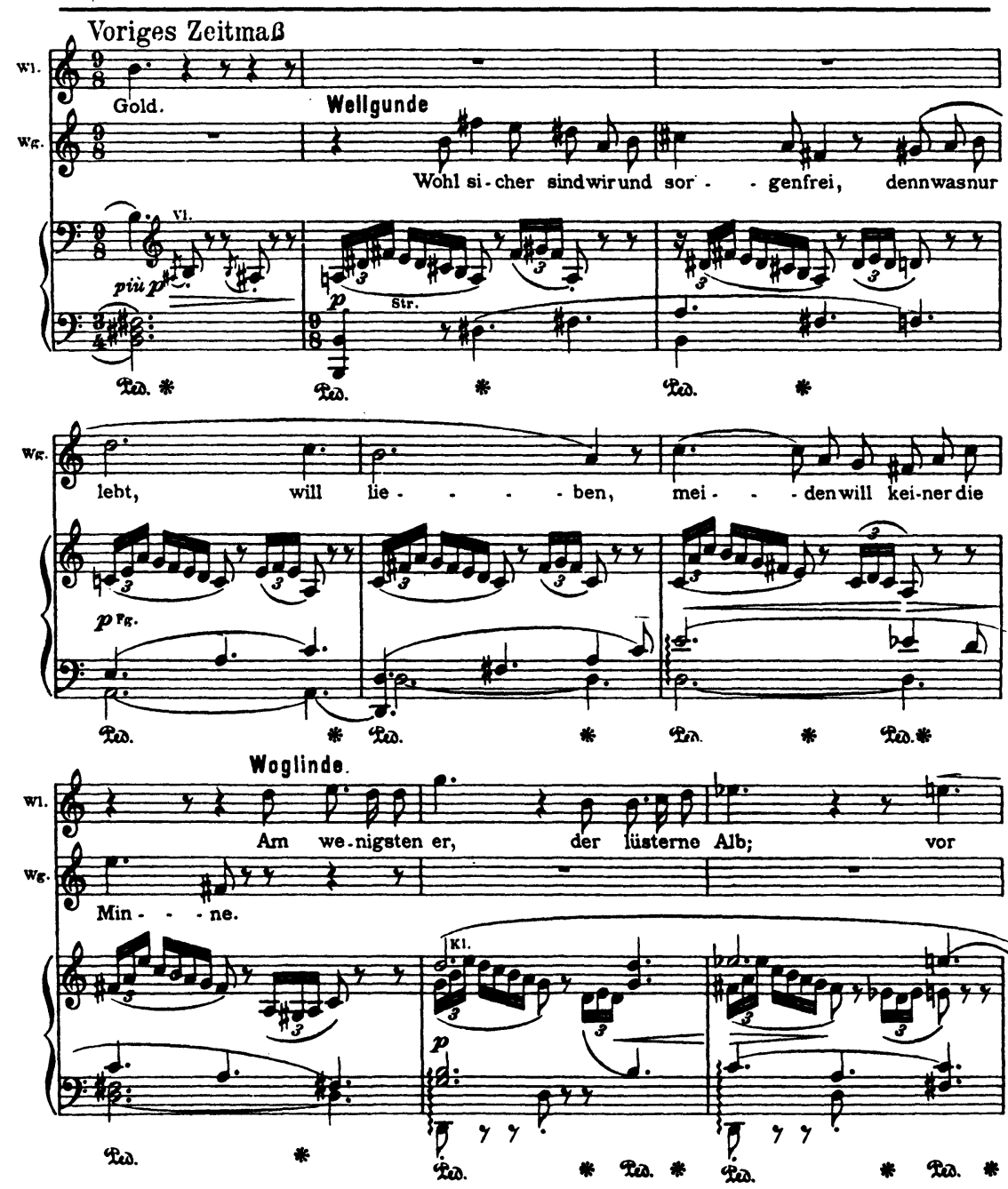

Figure 26. : O.R., I, 51 
'Wohl sicher sind wir/und sorgen frei'49 : il faut donner à chaque mot toute son attention. Puis, Wagner a indiqué un grand legato : ce qu'elle dit là - 'tout ce qui vit aimera' - les filles le croient. Mais en même temps, avec une certaine exagération. Les legati sont rares dans L'Or du Rhin et ils traduisent le plus souvent une sensualité vraie. Puis, avec 'meiden will Keiner die Minne,' 50 le ton change encore et je crois qu'il faut insister sur le $k$ de 'keiner'.

Je pense encore à ce passage (O.R. I, 27-8)), plus tôt, où Flosshilde mène Alberich en bateau : conformément à ce qu'indique Wagner, j'ai cherché à obtenir cette différence entre 'Seligster Mann !'51 et 'Wärst $\mathrm{du}$ mir hold's2 qui doit être d'autant plus piano qu'après ces legati, on tombe dans quelque chose de très détaché, dur et méchant. Après l'évocation de sa 'Krötengestalt's3 et de sa 'Stimme Gekrächz's4 où il faut accentuer les sonorités rauques, on retrouve un piano legato très différent ('O dürft ich staunend und stumm/sie nur hören und sehn!'55 où l'ironie et la méchanceté se mêlent à l'étonnement : comment peut-on être si laid !

J.J.N. : Mais ce principe de différenciation se rencontre surtout dans la distinction entre les personnages?

J.T. : Oui, c'est quelque chose de très important dans la mise en scène de Chéreau : les trois filles du Rhin, les deux géants, les neuf Walkyries

\footnotetext{
49 'Ainsi sommes-nous sans crainte / et sans soucis'.

50 'Nul ne veut fuir l'amour'.

51 'O le plus heureux des hommes !'

52 'Si tu m'étais favorable !'

53 'tes formes de crapaud'.

54 'le croassement de ta voix'.

s5 'Oh ! Puissé-je, étonnée et muette, / n'entendre et ne voir qu'eux !'
} 
et les trois Nornes ne sont pas traités comme des masses indifférenciées, mais comme des groupes au sein desquels chaque personnage conserve son individualité propre. Cela apparaît dans sa régie. Mon travail a consisté à trouver des traits d'individualité au niveau vocal.

\section{J.J.N. : Ca vous a été difficile?}

J.T. : Pas vraiment, parce que c'était déjà mon principe. Je fais ça ailleurs. Et c'est ce qui me fascine chez Wagner. Chanter Verdi, c'est le même problème que danser Le Lac des cygnes. Mais chanter Wagner, c'est comme danser L'Oiseau de feu. A cet égard, Wagner, c'est le début de la modernité : les expressions sont réelles, et ça m'intéresse beaucoup d'orienter mon travail pour rendre sensible ce qui est présent.

\section{J.J.N. : Exemple?}

J.T. : Pour les filles du Rhin, je crois que Woglinde se veut très séductrice. 'Nun nahe dich mir!'s6 (O.R., I, 16): je lui fais accentuer et prolonger le $\mathrm{m}$ : il y a de l'ironie mais aussi de la séduction. Et on a vu tout à l'heure que Flosshilde était beaucoup plus prudente. Fasolt, on le sait, est beaucoup plus romantique que son frère. Cela se sent dans le contour mélodique, dans ses legati. Pour ce qui est des Walkyries, j'avais remarqué, dans les esquisses publiées par Westernhagen (1973 : 132-3), que Waltraute avait été conçue un peu à part de ses soeurs, ce qui n'est pas étonnant puisque c'est elle qui viendra auprès de Brünnhilde en ambassadrice dans le Crépuscule. Regardez l'écriture :

56 'A présent, approche-toi de moi !' 

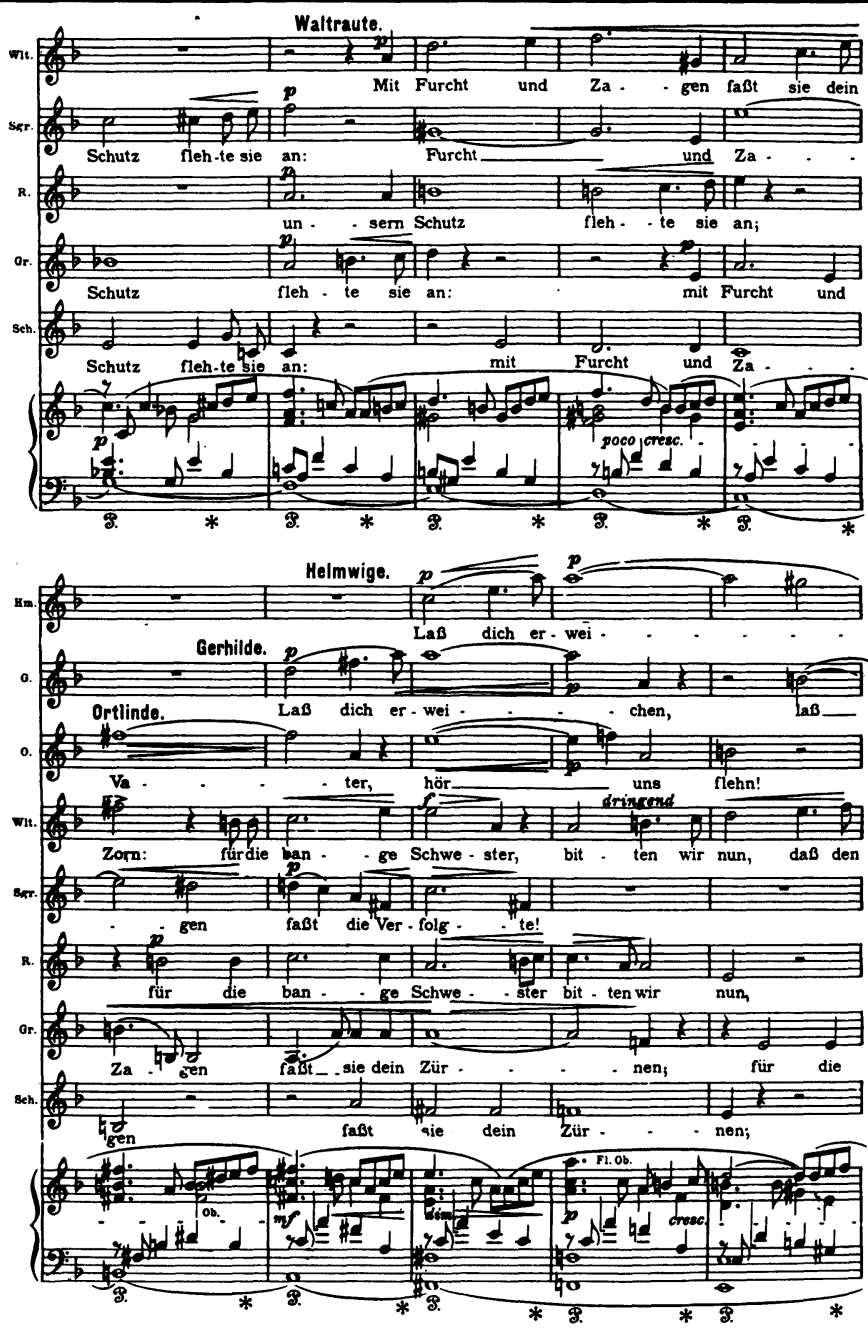

Figure 27. : W., III, 263

Tous les soprani restent piano, mais Waltraute a un piano crescendo, puis un forte. 
J.J.N. : Pensez-vous qu'on puisse le remarquer?

J.T. : On l'entend, je crois, et on le voit : j'ai signalé la chose à Chéreau et il s'est servi de l'idée pour donner sur la scène beaucoup plus d'autonomie à Waltraute. Je crois que c'est un grand moment de correspondance entre la mise en scène et la musique, et qui vient de l'écriture. Dans toute cette scène, $j$ 'ai essayé de faire respecter toutes les variations de dynamique et ça a été relativement facile parce que j'ai eu les mêmes chanteuses au cours des trois dernières années.

J.J.N. : Et les filles du Rhin du Crépuscule ?

J.T. : Vous retrouvez la même finesse d'écriture. Voyez ce petit détail :

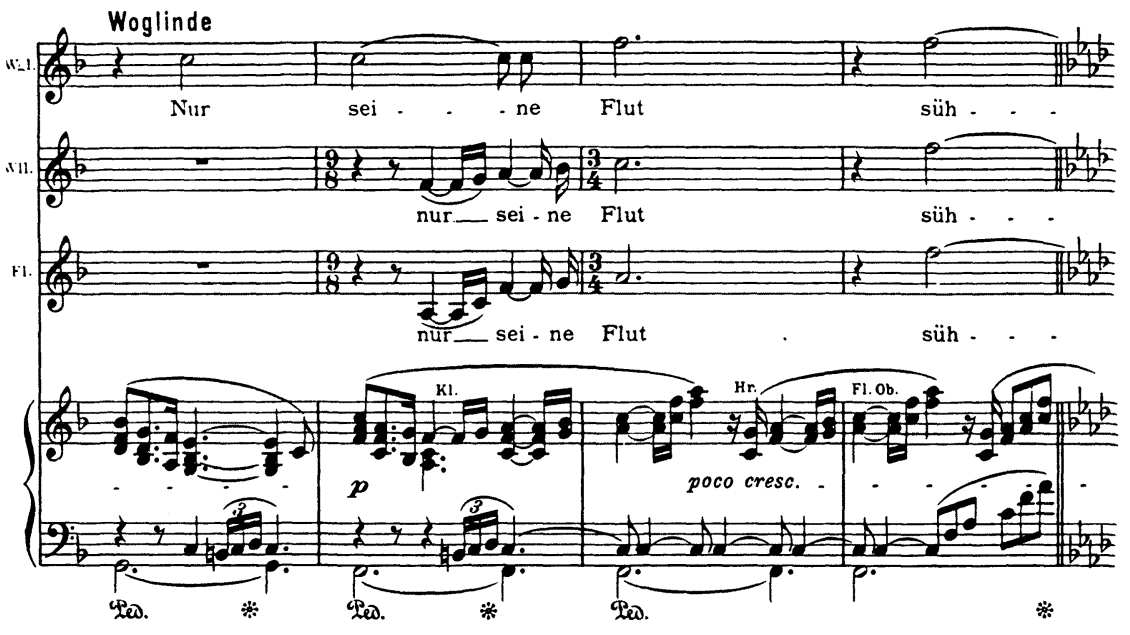

Figure 28. : C.D., III, 284 
Il faut que le 'nur' de Woglinde soit plus fort que son 'seine', car sur ce mot, les deux autres filles vont à leur tour chanter 'nur seine', et il faut qu'on les entende.

J'aime beaucoup cet autre passage de la même scène qui est presque viennois :
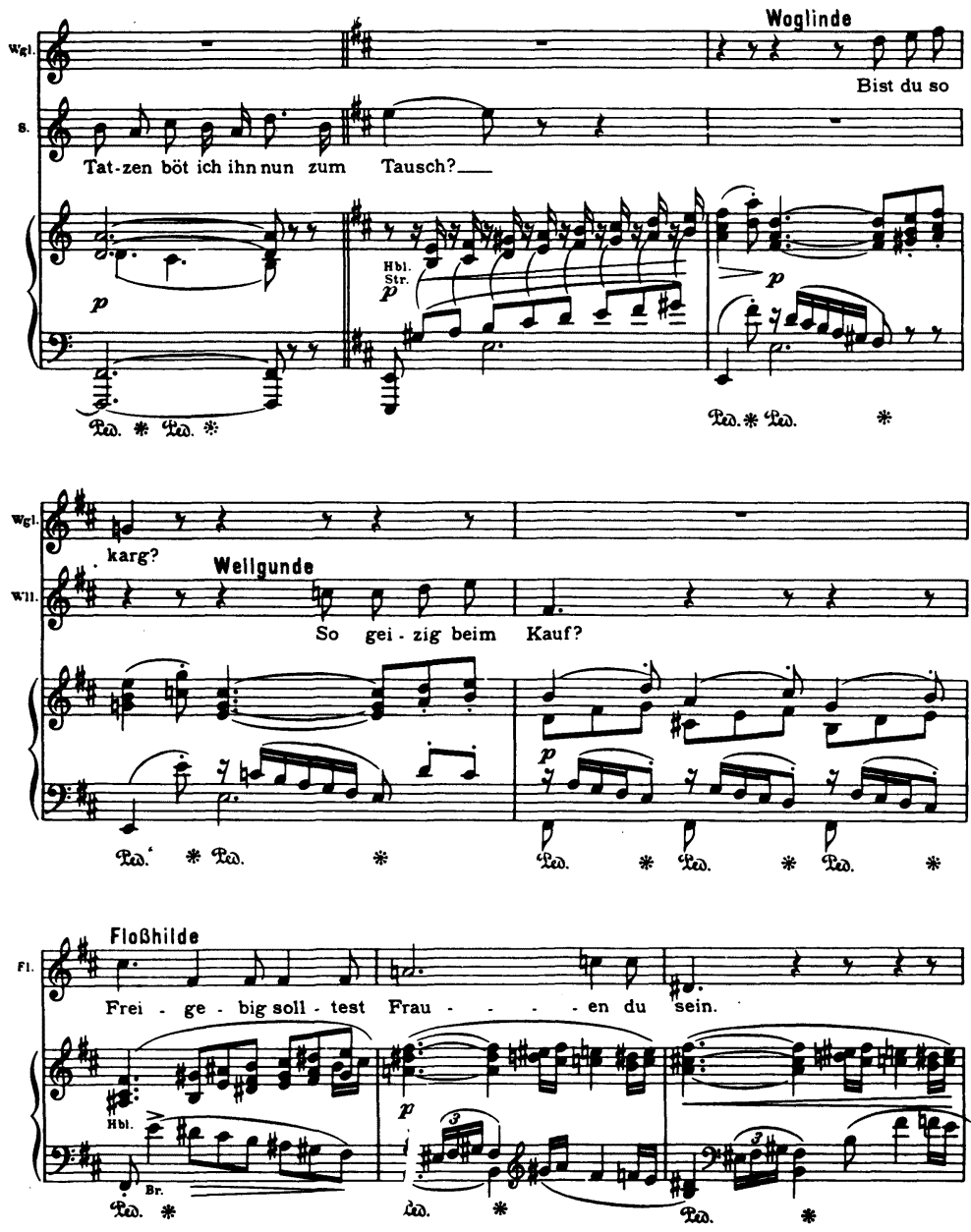

Figure 29. : C.D., III, 273-4 
On a trois portamenti successifs : 'so karg', 'beim Kauf', 'du sein'. Ca fait 'Pierrot Lunaire', mais aussi fin de siècle. Je pense aux valses de Mahler et je crois qu'il faut exploiter le côté sucré de la scène. D'autant plus qu'à la page suivante, c'est terrible, les filles sont usées, il n'y a plus d'ironie : 'Sie ist wohl schlimm?/Sie schlägt dich wohl?/Ihre Hand fühlt schon der Held.'57

Après l'intervention de Siegfried, elles vont à nouveau se moquer de lui :

57 'Est-elle si méchante ? / Elle te bat peut-être ? / Le héros veut déjà sa main !' 


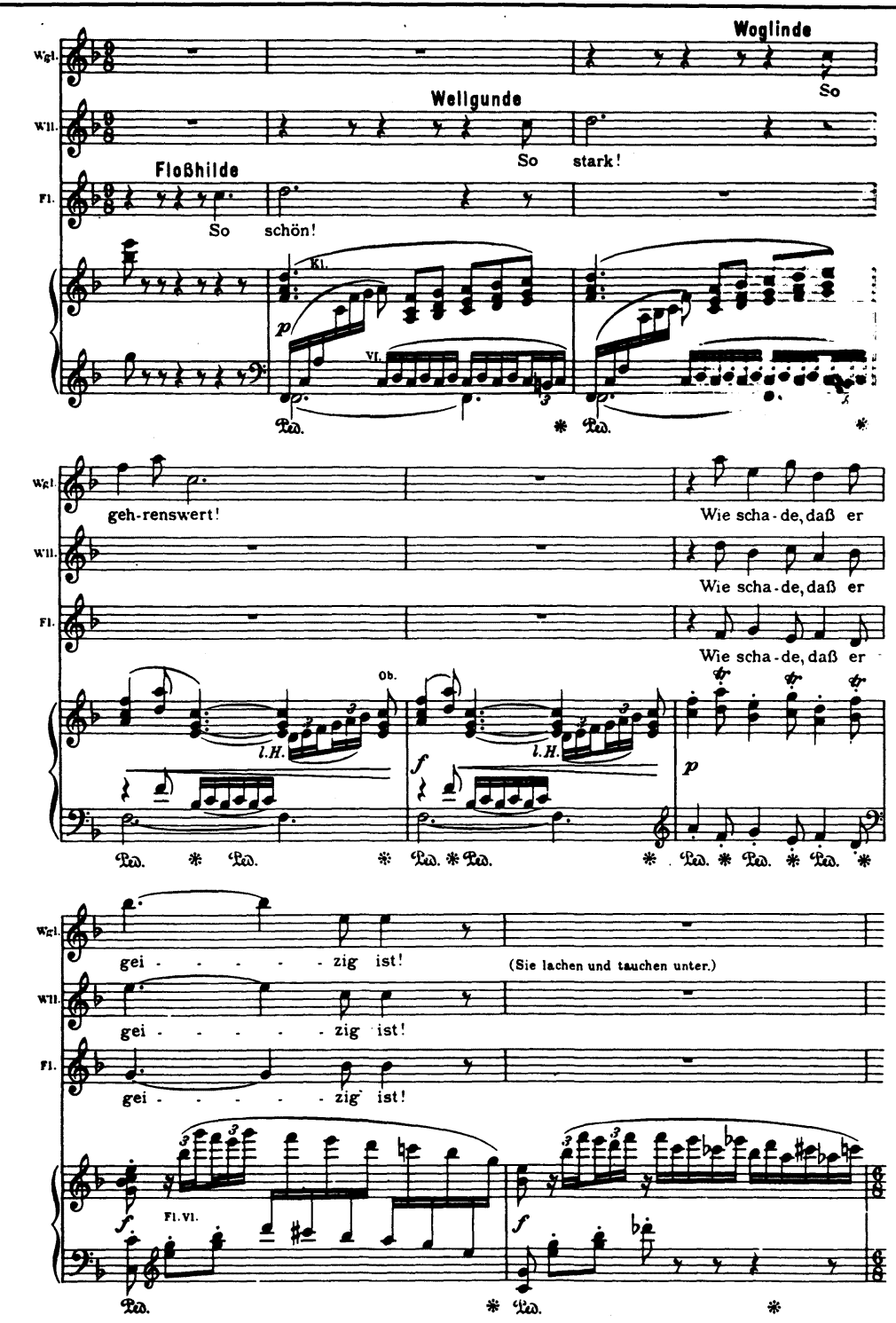

Figure 30. : C.D., III, 276-7 
Bien que Wagner n'ait rien écrit, j'essaie de différencier les trois mots 'schön', 'stark' et 'gehrenswert'. ${ }^{58}$ Le premier, en le faisant prononcer sur le ton de la séduction. Le second, un peu plus fort : chez Wagner, la croche fait la différence. Quant au troisième, il est dit à la fois de manière séduisante et ironique. Dans le passage d'ensemble qui suit, ça doit être acide et pointu, comme les staccati de l'orchestre, avec des voyelles très claires.

J.J.N. : Je crois que votre travail de différenciation des ensembles montre bien comment on peut retrouver au niveau vocal le travail de détail qu'on observe, chez Boulez au niveau de l'orchestre, et chez Chéreau au niveau de la mise en scène. Vous-même, si vous aviez à caractériser la rencontre entre la conception de Boulez et celle de Chéreau, que proposeriez-vous?

J.T. : Curieusement, je dirais que la rencontre se fait au niveau esthétique. Sur le plan du 'comment faire', de la qualité, de la justesse de ton, Chéreau cherche de son côté les gestes précis, l'atmosphère adéquate, l'expression appropriée, et c'est aussi ce que cherche Boulez avec son orchestre. C'est un Ring esthétique en ce sens que tous deux sont allés très loin dans la recherche des raffinements contenus dans l'oeuvre et qu'ils ont tenté, avec succès je crois, de révéler cette finesse grâce aux moyens d'expression qui leur sont propres.

58 'beau', 'fort', 'désirable'. 


\section{RÉFÉRENCES}

BOUlEZ, P., TATE, J., NATTIEZ, J. - J.,

1982 : Entretiens sur la Tétralogie du Centenaire. Montréal : Groupe de Recherches en Sémiologie Musicale, Publication interne No. 8.

NATTIEZ, J. - J.,

1983 : Tétralogies (Wagner, Boulez, Chéreau), essai sur l'infidélité. Paris : Christian Bourgois.

VON WESTERNHAGEN, C.,

1973 : Die Entstehung des "Ring”. Zürich et Freiburg : Atlantis.

Nous remercions les Editions Peters d'avoir aimablement autorisé la reproduction de tous les extraits musicaux figurant dans cet article. (N.D.E.) 